Article

\title{
Diversity-Oriented Synthesis Based on the DPPP-Catalyzed Mixed Double-Michael Reactions of Electron-Deficient Acetylenes and $\beta$-Amino Alcohols
}

\section{Yi Chiao Fan and Ohyun Kwon *}

Department of Chemistry and Biochemistry, University of California, Los Angeles, CA 90095, USA

* Author to whom correspondence should be addressed; E-Mail: ohyun@chem.ucla.edu.

Received: 6 April 2011; in revised form: 29 April 2011 / Accepted: 5 May 2011 /

Published: 5 May 2011

\begin{abstract}
In this study, we prepared oxizolidines through 1,3-bis(diphenylphosphino)propane (DPPP)-catalyzed mixed double-Michael reactions of $\beta$-amino alcohols with electron-deficient acetylenes. These reactions are very suitable for the diversity-oriented parallel syntheses of oxizolidines because: (i) they are performed under mild metal-free conditions and (ii) the products are isolated without complicated work-up. To demonstrate the applicability of mixed double-Michael reactions for the preparation of five-membered-ring heterocycles, we prepared 60 distinct oxazolidines from five $\beta$-amino alcohols and 12 electron-deficient acetylenes. We synthesized 36 of these 60 oxazolidines in enantiomerically pure form from proteinogenic amino acid-derived $\beta$-amino alcohols.
\end{abstract}

Keywords: phosphine catalysis; Michael reaction; oxazolidines; $\beta$-amino alcohols; acetylenes

\section{Introduction}

Because small organic heterocyclic compounds are found in most pharmaceuticals and agrochemicals and also in numerous natural products, additives, modifiers, and polymers [1,2], their construction remains of great interest to the synthetic organic chemistry community. In particular, the preparation of libraries of heterocyclic compounds through parallel combinatorial synthesis $[3,4]$ or branched diversity-oriented synthesis [5-7] would be an attractive approach toward identifying biologically active small organic molecules [8-10]. Simple, robust, and metal-free methods for 
synthesizing small molecules are especially attractive if these compounds are to be used for biological testing. Furthermore, reactions involving organocatalysis are becoming increasingly desirable in terms of their low environmental impact. From these considerations, phosphine-catalyzed annulation has served as an ideal format for synthesizing diverse groups of heterocyclic small molecules with high efficiency and minimal impact on the environment [11-29]. In particular, 1,3-bis(diphenylphosphino)propane (DPPP)-catalyzed double Michael addition has proved to be an atom-economical platform for preparing ten unique heterocyclic compounds [30,31].

A little over three decades ago, White and Baizer reported the first phosphine-catalyzed Michael additions of benzyl alcohol to activated olefins [32]. Only in the last two decades, however, did other synthetic research groups start studying Michael additions using nucleophilic phosphine catalysts and activated acetylenes. Inanaga demonstrated the first Michael additions of benzyl alcohol to activated acetylenes in the presence of nucleophilic phosphine catalysts [33]. When acetylenes are used as Michael acceptors, the Michael products possess one remaining degree of unsaturation, thereby enabling further incorporation of nucleophiles.

Capitalizing on the possibility of performing two consecutive Michael additions to activated acetylenes, Grossman elegantly demonstrated the double-Michael additions of carbon pro-nucleophiles to generate functionalized cyclohexanes under the influence of phosphine catalysts and bases [34,35]. Subsequently, Yavari employed catechol—an oxygen di-nucleophile — as the Michael donor [36]; although the yield of the resulting 1,3-benzodioxole was low (20\%, isolated), he confirmed that heteroatom double-Michael addition under phosphine catalysis was possible [37]. Nevertheless, to this day, few heterocyclic compounds have been generated using double-Michael additions.

To overcome poor efficiency and the inability to incorporate other heteroatoms such as nitrogen and sulfur, in the phosphine-catalyzed double-Michael reactions, we set out to develop a route to generate various heterocyclic compounds under general and robust phosphine catalysis conditions. By employing DPPP as the catalyst, we have synthesized functionalized oxazolidines, thiazolidines, and pyrrolidines in high efficiency (Scheme 1) [30]. By employing dinucleophiles tethered through aromatic rings, we have obtained indolines, dihydropyrrolopyridines, benzimidazolines, tetrahydroquinolines, tetrahydroisoquinolines, dihydrobenzo-1,4-oxazines, and dihydrobenzo-3,1oxazines using our developed mixed double-Michael strategy [31].

Scheme 1. DPPP-Catalyzed Mixed Double-Michael Reactions.

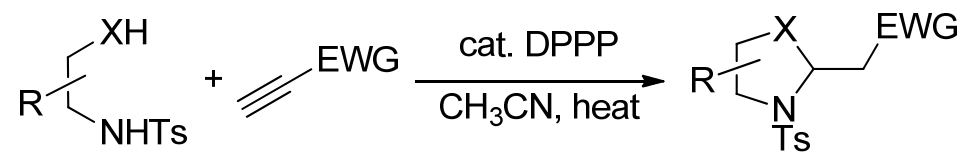

$\mathrm{X}=$ oxygen, sulfur, and carbon pro-nucleophiles

EWG = electron withdrawing group

Focusing on oxazolidines, here we report the synthesis of a library of 60 distinct oxazolidines through our mixed double-Michael strategy. Based on previously reported mixed double-Michael reactions [30,31], we knew that oxazolidines could be prepared from amino acid-derived pro-nucleophiles and electron-deficient acetylenes using DPPP as the catalyst. With such precedents in mind, here we expanded the reaction scope of the mixed double-Michael reaction to incorporate 
various oxygen-and-nitrogen-containing pro-nucleophiles and electron-deficient acetylenes. Under the reported conditions, we obtained the various oxazolidine derivatives rapidly and with high efficiency, enabling their future application in biological assays.

Oxazolidine, a five-membered-ring heterocycle containing both oxygen and nitrogen atoms, appears in many natural products that possess important biological activities. Quinocarcin and tetrazomine are two examples of the many naturally occurring oxazolidine-containing molecules displaying useful pharmacological activities (e.g., antitumor, cytotoxic, anti-inflammatory, and analgesic properties) [38,39]. Furthermore, enantiomerically pure oxazolidines can be used as chiral auxiliaries to induce asymmetry in organic reactions [40-45].

Figure 1. Oxazolidine-Containing Natural Products.

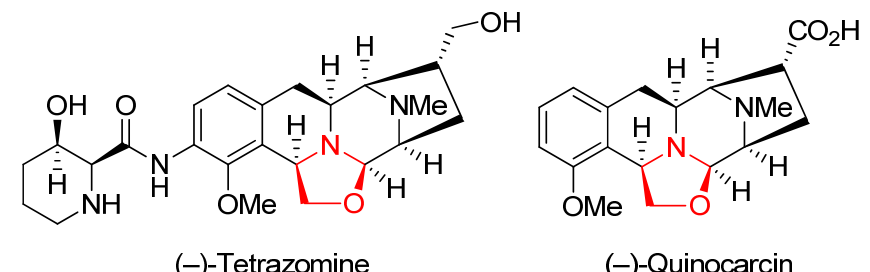

\section{Results and Discussion}

\subsection{Preparation of Pro-Nucleophiles}

The $\beta$-amino alcohol pro-nucleophiles that we used in the mixed double-Michael reactions were derived from natural L-amino acids and racemic cyclohexene oxide. We prepared the pro-nucleophiles derived from L-leucine, L-alanine, L-phenylalanine, and L-serine efficiently according to protocols described by Moberg and Craig [46]; first, we protected the amino groups of L-leucine, L-alanine, L-phenylalanine, and L-serine with $p$-toluenesulfonyl (tosyl) chloride and then we reduced their carboxylic acid units using lithium aluminum hydride [47,48]. We prepared the 2-aminocyclohexanolderived pro-nucleophile 1e from racemic cyclohexene oxide in three steps: opening of the epoxide with sodium azide, reduction of the azido group with palladium on charcoal $[49,50]$, and then protection of the amino group with tosyl chloride [51].

Scheme 2. Amino-Alcohol Pro-Nucleophiles.
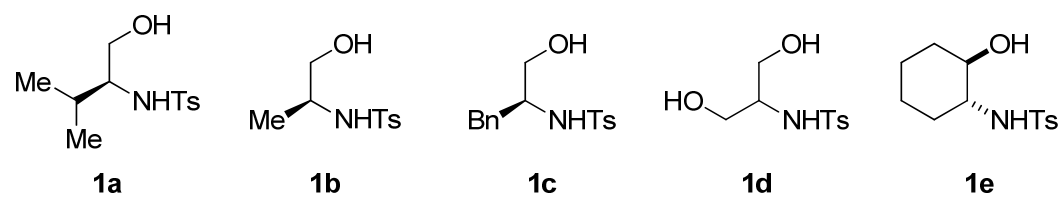

\subsection{Preparation of Electron-Deficient Acetylenes}

Table 1 lists the electron-deficient acetylenes that we prepared from corresponding aldehydes. Although the syntheses of the acetylenes 2a, 2c, and $2 \mathbf{e}$ have been reported previously, the acetylenes 2b, 2d, and $\mathbf{2 f}$ were unknown. Employing the protocol established by Oyelere and Calieno [52,53], we obtained each of these propargyl ketones in good yield after: (i) treating the pertinent aldehyde with 
ethynylmagnesium bromide at $0{ }^{\circ} \mathrm{C}$, slowly warming to ambient temperature, and then working-up the mixture after $4 \mathrm{~h}$ and (ii) oxidizing the resulting propargyl alcohol, without further purification, using Jones reagent.

Table 1. Synthesis of Electron-Deficient Acetylenes.

\begin{tabular}{|c|c|c|c|}
\hline Entry & $\mathbf{R}^{2}$ & Acetylene & Yield (\%) \\
\hline 1 & $\mathrm{Ph}$ & $2 \mathbf{a}$ & 83 \\
\hline 2 & $m, p-\mathrm{Cl}_{2} \mathrm{C}_{6} \mathrm{H}_{3}$ & $2 \mathbf{b}$ & 76 \\
\hline 3 & $m, p-\left(\mathrm{CH}_{3} \mathrm{O}\right)_{2} \mathrm{C}_{6} \mathrm{H}_{3}$ & 2c & 89 \\
\hline 4 & $p-\mathrm{FC}_{6} \mathrm{H}_{4}$ & 2d & 76 \\
\hline 5 & 1-naphthyl & $2 e$ & 67 \\
\hline 6 & 2-thienyl & $2 f$ & 75 \\
\hline
\end{tabular}

\subsection{Synthesis of an Oxazolidine Library}

With all the pro-nucleophiles and acetylenes at hand, we rapidly synthesized the desired chemical library of 60 distinct oxazolidines using DPPP as the catalyst (Tables 2-4). The oxazolidine library contained a diverse array of functional groups and provided several potential probes for examining various biological mechanisms.

The pro-nucleophiles 1a-c generated their desired oxazolidines in good to high yields, revealing that the reactions had good tolerance for various alkyl groups on the pro-nucleophiles (Table 2, entries 1-33). In contrast, the pro-nucleophile 1d provided its products in low to moderate yields, suggesting that the additional free hydroxyl functionality impeded the mixed double-Michael reactions (Table 2, entries 34-44). The yields of oxazolidines were even lower when we used 1e as the pro-nucleophile (Table 3), presumably because of steric hindrance about the secondary alcohol pro-nucleophile.

We tested various activated acetylenes to evaluate their reactivities in the mixed double-Michael reactions. Alkyl propiolates afforded their desired oxazolidines in good to high yields (Table 1, entries $1-3,12-14,23-25$, and 34-36). In contrast, the use of phenyl propiolate provided drastically lower yields of its oxazolidines (Table 1, entries 4, 15, 26, and 37), possibly because it was prone to hydrolysis under the reaction conditions.

Butynone and aromatic propargyl ketones afforded their oxazolidines in good yields from a range of pro-nucleophiles. Halogens and electron-donating groups on the benzene rings of the aromatic propargyl ketones were compatible with the mixed double-Michael reactions. Naphthyl and 2-thienyl groups were also tolerated in the reaction. Aside from using carbonyl moieties as electron withdrawing groups, we also employed an aromatic sulfone unit to activate the acetylene; tosyl acetylene provided its oxazolidines in good yields from the mixed double-Michael reactions (Table 4). 
Table 2. Synthesis of Trisubstituted Oxazolidines.

\begin{tabular}{|c|c|c|c|c|}
\hline Entry & $\mathbf{R}^{1}$ & $\overline{\mathbf{R}^{2}}$ & Oxazolidine & Yield (\%) \\
\hline 1 & $i-\operatorname{Pr}$ & $\mathrm{OMe}$ & $(2 S, 4 S)-\mathbf{3 a}$ & 59 \\
\hline 2 & $i$-Pr & OEt & $(2 S, 4 S)-\mathbf{3 b}$ & 75 \\
\hline 3 & $i$-Pr & $\mathrm{OBn}$ & $(2 S, 4 S)-3 \mathbf{c}$ & 63 \\
\hline 4 & $i$-Pr & $\mathrm{OPh}$ & $(2 S, 4 S)-\mathbf{3 d}$ & 22 \\
\hline 5 & $i$-Pr & $\mathrm{Me}$ & $(2 S, 4 S)-3 \mathbf{e}$ & 53 \\
\hline 6 & $i$-Pr & $\mathrm{Ph}$ & $(2 S, 4 S)-\mathbf{3 f}$ & 55 \\
\hline 7 & $i$-Pr & $m, p-\mathrm{Cl}_{2} \mathrm{C}_{6} \mathrm{H}_{3}$ & $(2 S, 4 S)-3 \mathbf{g}$ & 28 \\
\hline 8 & $i-\operatorname{Pr}$ & $m, p-\left(\mathrm{CH}_{3} \mathrm{O}\right)_{2} \mathrm{C}_{6} \mathrm{H}_{3}$ & $(2 S, 4 S)-3 \mathbf{h}$ & 58 \\
\hline 9 & $i-\operatorname{Pr}$ & $p-\mathrm{FC}_{6} \mathrm{H}_{4}$ & $(2 S, 4 S)-3 \mathbf{i}$ & 63 \\
\hline 10 & $i-\operatorname{Pr}$ & 1-naphthyl & $(2 S, 4 S)-3 \mathbf{j}$ & 70 \\
\hline 11 & $i-\operatorname{Pr}$ & 2-thienyl & $(2 S, 4 S)-3 \mathbf{k}$ & 37 \\
\hline 12 & $\mathrm{Me}$ & $\mathrm{OMe}^{\circ}$ & $(2 S, 4 S)-\mathbf{4 a}$ & 68 \\
\hline 13 & $\mathrm{Me}$ & OEt & $(2 S, 4 S)-\mathbf{4 b}$ & 68 \\
\hline 14 & $\mathrm{Me}$ & OBn & $(2 S, 4 S)-\mathbf{4 c}$ & 64 \\
\hline 15 & $\mathrm{Me}$ & $\mathrm{OPh}$ & $(2 S, 4 S)-\mathbf{4 d}$ & 14 \\
\hline 16 & $\mathrm{Me}$ & $\mathrm{Me}$ & $(2 S, 4 S)-4 \mathbf{e}$ & 68 \\
\hline 17 & $\mathrm{Me}$ & $\mathrm{Ph}$ & $(2 S, 4 S)-\mathbf{4 f}$ & 57 \\
\hline 18 & $\mathrm{Me}$ & $m, p-\mathrm{Cl}_{2} \mathrm{C}_{6} \mathrm{H}_{3}$ & $(2 S, 4 S)-\mathbf{4 g}$ & 41 \\
\hline 19 & $\mathrm{Me}$ & $m, p-\left(\mathrm{CH}_{3} \mathrm{O}\right)_{2} \mathrm{C}_{6} \mathrm{H}_{3}$ & $(2 S, 4 S)-4 \mathbf{h}$ & 90 \\
\hline 20 & $\mathrm{Me}$ & $p-\mathrm{FC}_{6} \mathrm{H}_{4}$ & $(2 S, 4 S)-4 \mathbf{i}$ & 27 \\
\hline 21 & $\mathrm{Me}$ & 1-naphthyl & $(2 S, 4 S)-\mathbf{4 j}$ & 33 \\
\hline 22 & $\mathrm{Me}$ & 2-thienyl & $(2 S, 4 S)-\mathbf{4 k}$ & 25 \\
\hline 23 & $\mathrm{Bn}$ & $\mathrm{OMe}^{\circ}$ & $(2 S, 4 S)-\mathbf{5 a}$ & 66 \\
\hline 24 & $\mathrm{Bn}$ & OEt & $(2 S, 4 S)-5 \mathbf{b}$ & 81 \\
\hline 25 & $\mathrm{Bn}$ & $\mathrm{OBn}$ & $(2 S, 4 S)-5 \mathbf{c}$ & 76 \\
\hline 26 & $\mathrm{Bn}$ & $\mathrm{OPh}$ & $(2 S, 4 S)-5 \mathbf{d}$ & 5 \\
\hline 27 & $\mathrm{Bn}$ & $\mathrm{Me}$ & $(2 S, 4 S)-5 \mathbf{e}$ & 45 \\
\hline 28 & $\mathrm{Bn}$ & $\mathrm{Ph}$ & $(2 S, 4 S)-\mathbf{5 f}$ & 71 \\
\hline 29 & $\mathrm{Bn}$ & $m, p-\mathrm{Cl}_{2} \mathrm{C}_{6} \mathrm{H}_{3}$ & $(2 S, 4 S)-5 \mathbf{g}$ & 30 \\
\hline 30 & $\mathrm{Bn}$ & $m, p-\left(\mathrm{CH}_{3} \mathrm{O}\right)_{2} \mathrm{C}_{6} \mathrm{H}_{3}$ & $(2 S, 4 S)-5 \mathbf{h}$ & 98 \\
\hline 31 & $\mathrm{Bn}$ & $p-\mathrm{FC}_{6} \mathrm{H}_{4}$ & $(2 S, 4 S)-5 \mathbf{i}$ & 51 \\
\hline 32 & $\mathrm{Bn}$ & 1-naphthyl & $(2 S, 4 S)-\mathbf{5 j}$ & 96 \\
\hline 33 & $\mathrm{Bn}$ & 2-thienyl & $(2 S, 4 S)-5 \mathbf{k}$ & 41 \\
\hline 34 & $\mathrm{CH}_{2} \mathrm{OH}$ & $\mathrm{OMe}$ & $( \pm)-6 \mathbf{a}$ & 38 \\
\hline 35 & $\mathrm{CH}_{2} \mathrm{OH}$ & OEt & $( \pm)-6 b$ & 50 \\
\hline 36 & $\mathrm{CH}_{2} \mathrm{OH}$ & OBn & $( \pm)-6 c$ & 37 \\
\hline 37 & $\mathrm{CH}_{2} \mathrm{OH}$ & $\mathrm{OPh}$ & $( \pm)-6 d$ & 8 \\
\hline 38 & $\mathrm{CH}_{2} \mathrm{OH}$ & $\mathrm{Me}$ & $( \pm)-6 e$ & 29 \\
\hline 39 & $\mathrm{CH}_{2} \mathrm{OH}$ & $\mathrm{Ph}$ & $( \pm)-6 f$ & 23 \\
\hline 40 & $\mathrm{CH}_{2} \mathrm{OH}$ & $m, p-\mathrm{Cl}_{2} \mathrm{C}_{6} \mathrm{H}_{3}$ & $( \pm)-6 g$ & 12 \\
\hline 41 & $\mathrm{CH}_{2} \mathrm{OH}$ & $m, p-\left(\mathrm{CH}_{3} \mathrm{O}\right)_{2} \mathrm{C}_{6} \mathrm{H}_{3}$ & $( \pm)-6 h$ & 38 \\
\hline 42 & $\mathrm{CH}_{2} \mathrm{OH}$ & $p-\mathrm{FC}_{6} \mathrm{H}_{4}$ & $( \pm)-6 \mathbf{i}$ & 23 \\
\hline 43 & $\mathrm{CH}_{2} \mathrm{OH}$ & 1-naphthyl & $( \pm)-6 \mathbf{j}$ & 24 \\
\hline 44 & $\mathrm{CH}_{2} \mathrm{OH}$ & 2-thienyl & $( \pm)-6 k$ & 58 \\
\hline
\end{tabular}


Table 3. Synthesis of Octahydrobenzoxazolidines.

\begin{tabular}{|c|c|c|c|}
\hline Entry & $\mathbf{R}$ & Oxazolidine & Yield (\%) \\
\hline 1 & $\mathrm{OMe}$ & $( \pm)-7 a$ & 15 \\
\hline 2 & $\mathrm{OEt}$ & $( \pm)-7 \mathbf{b}$ & 37 \\
\hline 3 & OBn & $( \pm)-7 c$ & 19 \\
\hline 4 & $\mathrm{OPh}$ & $( \pm)-7 d$ & 13 \\
\hline 5 & $\mathrm{Me}$ & $( \pm)-7 e$ & 33 \\
\hline 6 & $\mathrm{Ph}$ & $( \pm)-7 f$ & 32 \\
\hline 7 & $m, p-\mathrm{Cl}_{2} \mathrm{C}_{6} \mathrm{H}_{3}$ & $( \pm)-7 g$ & 18 \\
\hline 8 & $m, p-\left(\mathrm{CH}_{3} \mathrm{O}\right)_{2} \mathrm{C}_{6} \mathrm{H}_{3}$ & $( \pm)-7 h$ & 93 \\
\hline 9 & $p-\mathrm{FC}_{6} \mathrm{H}_{4}$ & $( \pm)-7 \mathbf{i}$ & 27 \\
\hline 10 & 1-naphthyl & $( \pm)-7 \mathbf{j}$ & 33 \\
\hline 11 & 2-thienyl & $( \pm)-7 \mathbf{k}$ & 25 \\
\hline
\end{tabular}

Table 4. Tosyl Acetylene as Mixed Double-Michael Acceptor.

\begin{tabular}{|c|c|c|c|}
\hline Entry & Pro-Nucleophile & Oxazolidine & Yield (\%) \\
\hline 1 & & $(2 S, 4 S)-\mathbf{8 a}$ & 30 \\
\hline 2 & & $(2 S, 4 S)-\mathbf{8 b}$ & 68 \\
\hline 3 & & $(2 S, 4 S)-\mathbf{8 c}$ & 65 \\
\hline 4 & & $( \pm)-8 d$ & 81 \\
\hline 5 & & $( \pm)-8 e$ & 37 \\
\hline
\end{tabular}

\section{Experimental}

\subsection{General}

All reactions were performed in flamed-dried or oven-dried round-bottom flasks, Schlenk flasks, or two-neck flasks. A glass water condenser, fitted with a rubber septum, was attached to each flask. All reactions were performed under a positive pressure of argon. A syringe pump and stainless-steel needles were used to inject the acetylene derivatives into the refluxing reaction mixtures. Reactions were monitored through thin-layer chromatography (TLC) on $0.25-\mathrm{mm}$ SiliCycle silica gel plates. Plates were visualized under UV light or through $p$-anisaldehyde or potassium permanganate staining 
followed by heating $(<1 \mathrm{~min})$ with a heat gun. Flash column chromatography (FCC) was performed using SiliCycle Silica-P Flash silica gel $(60 \AA$ pore size, $40-63 \mu \mathrm{m})$. Organic solutions were concentrated using rotary evaporators.

\subsection{Materials and Reagents}

Reagents were used as received from commercial sources. Methyl propiolate and ethyl propiolate were purchased from TCI America. Tosyl acetylene and 3-butyn-2-one were purchased from Aldrich. Acetonitrile and dichloromethane were distilled from calcium hydride under a positive pressure of argon. Tetrahydrofuran was distilled from sodium and benzophenone under a positive pressure of argon.

\subsection{Instrumentation}

IR spectra were recorded using a Thermo Nicolet Avatar 370 FT-IR spectrometer. NMR spectra were recorded using Bruker ARX-400 instrument, calibrated to signals from the solvent as an internal reference [7.26 (residual $\left.\mathrm{CHCl}_{3}\right)$ and $77.00\left(\mathrm{CDCl}_{3}\right)$ ppm for ${ }^{1} \mathrm{H}$ - and ${ }^{13} \mathrm{C}-\mathrm{NMR}$ spectra, respectively]. Data for ${ }^{1} \mathrm{H}$ NMR spectra are reported as follows: chemical shift $(\delta, \mathrm{ppm})$, multiplicity, coupling constant $(\mathrm{Hz})$, and integration. Data for ${ }^{13} \mathrm{C}$-NMR spectra are reported in terms of chemical shift. The following abbreviations are used to denote the multiplicities: $\mathrm{s}=\operatorname{singlet} ; \mathrm{d}=$ doublet; $\mathrm{t}=$ triplet; $\mathrm{q}=$ quartet; $\mathrm{m}=$ multiplet. Mass spectra of the samples were recorded using a Waters LCT Premier XE time-of-flight instrument controlled by MassLynx 4.1 software. Samples were infused through direct loop injection from a Waters Acquity UPLC into the multi-mode ionization source. The lock mass standard for accurate mass determination was leucine enkephalin (Sigma L9133). An Agilent Technologies 5975 inert XL mass-selective detector GCMS was also used.

\subsection{Synthesis of Electron-Deficient Acetylenes}

1-Phenylprop-2-yn-1-one (2a) was prepared in 83\% yield using the protocol described by Oyelere and Calieno [52,53]. Spectral data matched those reported in the literature [53].

1-(3,4-Dichlorophenyl)prop-2-yn-1-one (2b) was prepared in 76\% yield using the protocol described by Oyelere and Calieno [52,53]; yellow solid; IR $\left(\mathrm{CH}_{2} \mathrm{Cl}_{2}\right) v_{\max } 3219.3,2093.63,1639.0,1579.0$, 1232.4, 750.1 cm ${ }^{-1}$; ${ }^{1} \mathrm{H}-\mathrm{NMR}\left(400 \mathrm{MHz}, \mathrm{CDCl}_{3}\right) \delta 8.23(\mathrm{~s}, 1 \mathrm{H}), 7.97-8.00(\mathrm{~m}, 1 \mathrm{H}), 7.58-7.61$ $(\mathrm{m}, 1 \mathrm{H}), 3.51(\mathrm{~s}, 1 \mathrm{H}) ;{ }^{13} \mathrm{C}-\mathrm{NMR}\left(100 \mathrm{MHz}, \mathrm{CDCl}_{3}\right) \delta 174.9,139.4,135.7,133.5,130.9,128.4,81.9$, 79.5, 77.2; GCMS (EI+) calcd for $\left[\mathrm{C}_{9} \mathrm{H}_{4} \mathrm{Cl}_{2} \mathrm{O}\right]: \mathrm{m} / z$ 199.0, found 199.0.

1-(3,4-Dimethoxyphenyl)prop-2-yn-1-one (2c): 89\% yield; spectral data matched those reported in the literature [54].

1-(4-Fluorophenyl)prop-2-yn-1-one (2d) was prepared in 76\% yield using the protocol described by Oyelere and Calieno [52,53]; brown solid; IR $\left(\mathrm{CH}_{2} \mathrm{Cl}_{2}\right) v_{\max } 3211.7,2093.45,1651.4,1598.3,1255.5$, 1155.4, 754.6 cm ${ }^{-1} ;{ }^{1} \mathrm{H}-\mathrm{NMR}\left(400 \mathrm{MHz}, \mathrm{CDCl}_{3}\right) \delta 8.20$ (d, J=4.0 Hz, 2H), 7.18 (d, $\left.J=4.0 \mathrm{~Hz}, 2 \mathrm{H}\right)$, 
3.45 (s, 1H); ${ }^{13} \mathrm{C}-\mathrm{NMR}\left(100 \mathrm{MHz}, \mathrm{CDCl}_{3}\right) \delta 175.7,168.0,165.4,132.5,116.1,115.9,81.0,80.0,77.2$; GCMS (EI+) calcd for $\left[\mathrm{C}_{9} \mathrm{H}_{5} \mathrm{FO}\right]: \mathrm{m} / z$ 148.1, found 148.1 .

1-(Naphthalen-1-yl)prop-2-yn-1-one (2e): 67\% yield; spectral data matched those reported in the literature [55].

1-(Thien-2-yl)prop-2-yn-1-one (2f) was prepared in 75\% yield using the protocol described by Oyelere and Calieno [52,53]; brown solid; IR $\left(\mathrm{CH}_{2} \mathrm{Cl}_{2}\right) v_{\max }$ 3239.2, 2093.3, 1619.1, 1409.8, 1279.2, $734.4 \mathrm{~cm}^{-1}$; ${ }^{1} \mathrm{H}-\mathrm{NMR}\left(400 \mathrm{MHz}, \mathrm{CDCl}_{3}\right) \delta 7.96(\mathrm{~d}, J=4.0 \mathrm{~Hz}, 1 \mathrm{H}), 7.74(\mathrm{~d}, J=4.0 \mathrm{~Hz}, 1 \mathrm{H}), 7.16(\mathrm{t}$, $J=4.0 \mathrm{~Hz}, 1 \mathrm{H}), 3.37(\mathrm{~s}, 1 \mathrm{H}) ;{ }^{13} \mathrm{C}-\mathrm{NMR}\left(100 \mathrm{MHz}, \mathrm{CDCl}_{3}\right) \delta 169.1,144.1,136.2,129.1,128.5,79.4$, 77.3; GCMS (EI+) calcd for $\left[\mathrm{C}_{7} \mathrm{H}_{4} \mathrm{OS}\right]: \mathrm{m} / z$ 136.2, found 136.1 .

Phenyl propiolate was prepared in $90 \%$ yield according to the protocol described by Ramachandran; spectral data matched those reported in the literature [56].

Benzyl propiolate was prepared in $90 \%$ yield according to the protocol described by Ramachandran; spectral data matched those reported in the literature [57].

\subsection{General Procedure for Mixed Double-Michael Reaction}

The $N$-tosylamido alcohol pro-nucleophile $(1 \mathrm{mmol})$, DPPP $(84.2 \mathrm{mg}, 0.2 \mathrm{mmol})$, and $\mathrm{CH}_{3} \mathrm{CN}$ $(5 \mathrm{~mL})$ were placed in a dried two-neck round-bottom flask $(25 \mathrm{~mL})$ equipped with a magnetic stirrer bar. The mixture was brought to reflux in an oil bath (external temperature: $90{ }^{\circ} \mathrm{C}$ ). A solution of the electron-deficient acetylene $(1.2 \mathrm{mmol})$ in $\mathrm{CH}_{3} \mathrm{CN}(1 \mathrm{~mL})$ was added into the flask over $1 \mathrm{~h}$ using a syringe pump. [In the case of ethynyl $p$-tolyl sulfone, the acetylene $(1.2 \mathrm{mmol})$ was added to the flask over $2 \mathrm{~h}$ via syringe pump.] The reaction mixture was stirred overnight under reflux, under a positive pressure of argon, monitoring with TLC (20\% EtOAc in hexanes). The resulting reaction mixture was concentrated in vacuo and purified by FCC on silica gel (20\% EtOAc in hexanes) to yield the cyclized product.

Methyl 2-[(2S,4S)-4-isopropyl-3-tosyloxazolidin-2-yl]acetate (3a). 59\% yield; white solid; IR $\left(\mathrm{CH}_{2} \mathrm{Cl}_{2}\right) v_{\max } 2960.7,1741.9,1598.1,1351.2,1164.7 \mathrm{~cm}^{-1} ;{ }^{1} \mathrm{H}-\mathrm{NMR}\left(400 \mathrm{MHz}, \mathrm{CDCl}_{3}\right) \delta 7.68(\mathrm{~d}$, $J=8.4 \mathrm{~Hz}, 2 \mathrm{H}), 7.29(\mathrm{~d}, J=8.0 \mathrm{~Hz}, 2 \mathrm{H}), 5.23(\mathrm{dd}, J=8.8,3.2 \mathrm{~Hz}, 1 \mathrm{H}), 3.69(\mathrm{dd}, J=9.2,2.4 \mathrm{~Hz}, 1 \mathrm{H})$, $3.64(\mathrm{~s}, 3 \mathrm{H}), 3.35$ (ddd, $J=7.9,6.1,2.1 \mathrm{~Hz}, 1 \mathrm{H}), 3.00-3.07(\mathrm{~m}, 2 \mathrm{H}), 2.62(\mathrm{dd}, J=15.8,8.6 \mathrm{~Hz}, 1 \mathrm{H})$, $2.36(\mathrm{~s}, 3 \mathrm{H}), 1.78-1.86(\mathrm{~m}, 1 \mathrm{H}), 0.95(\mathrm{~d}, J=6.8 \mathrm{~Hz}, 3 \mathrm{H}), 0.87(\mathrm{~d}, J=6.8 \mathrm{~Hz}, 3 \mathrm{H}) ;{ }^{13} \mathrm{C}-\mathrm{NMR}(100$ $\left.\mathrm{MHz}, \mathrm{CDCl}_{3}\right) \delta 169.9,144.4,133.7,130.0,127.9,88.8,67.8,64.9,51.8,41.8,31.3,21.5,19.5,18.4$; HRMS (ESI+) calcd for $\left[\mathrm{C}_{16} \mathrm{H}_{23} \mathrm{NO}_{5} \mathrm{~S}\right]^{+}: \mathrm{m} / z$ 342.1370, found 342.1364 .

Ethyl 2-[(2S,4S)-4-isopropyl-3-tosyloxazolidin-2-yl]acetate (3b). 75\% yield; yellow oil; IR $\left(\mathrm{CH}_{2} \mathrm{Cl}_{2}\right)$ $v_{\max }$ 2964.4, 1736.0, 1598.0, 1351.6, $1165.3 \mathrm{~cm}^{-1} ;{ }^{1} \mathrm{H}-\mathrm{NMR}\left(400 \mathrm{MHz}, \mathrm{CDCl}_{3}\right) \delta 7.65(\mathrm{~d}, J=8.4 \mathrm{~Hz}$, $2 \mathrm{H}), 7.25(\mathrm{~d}, J=8.0 \mathrm{~Hz}, 2 \mathrm{H}), 5.21(\mathrm{dd}, J=8.8,3.2 \mathrm{~Hz}, 1 \mathrm{H}), 4.07(\mathrm{q}, J=7.2 \mathrm{~Hz}, 2 \mathrm{H}), 3.66(\mathrm{dd}, J=9.0$, $2.2 \mathrm{~Hz}, 1 \mathrm{H}), 3.32$ (ddd, $J=7.7,6.1,1.9 \mathrm{~Hz}, 1 \mathrm{H}), 2.96-3.04(\mathrm{~m}, 2 \mathrm{H}), 2.58(\mathrm{dd}, J=15.8,8.6 \mathrm{~Hz}, 1 \mathrm{H})$, 
$2.32(\mathrm{~s}, 3 \mathrm{H}), 1.75-1.84(\mathrm{~m}, 1 \mathrm{H}), 1.17(\mathrm{t}, J=7.0 \mathrm{~Hz}, 3 \mathrm{H}), 0.92(\mathrm{~d}, J=6.8 \mathrm{~Hz}, 3 \mathrm{H}), 0.84(\mathrm{~d}, J=6.8 \mathrm{~Hz}$, $3 \mathrm{H}) ;{ }^{13} \mathrm{C}-\mathrm{NMR}\left(100 \mathrm{MHz}, \mathrm{CDCl}_{3}\right) \delta 169.5,144.3,133.7,130.0,127.9,88.9,67.8,64.8,60.7,42.0$, $31.3,21.4,19.5,18.4,14.1$; HRMS (ESI+) calcd for $\left[\mathrm{C}_{17} \mathrm{H}_{25} \mathrm{NO}_{5} \mathrm{~S}\right]^{+}: m / z 356.1526$, found 356.1518 .

Benzyl 2-[(2S,4S)-4-isopropyl-3-tosyloxazolidin-2-yl]acetate (3c). 63\% yield; yellow oil; $\mathrm{IR}\left(\mathrm{CH}_{2} \mathrm{Cl}_{2}\right)$ $v_{\max }$ 3031.4, 2962.6, 1738.7, 1597.8, 1351.2, $1164.7 \mathrm{~cm}^{-1} ;{ }^{1} \mathrm{H}-\mathrm{NMR}\left(400 \mathrm{MHz}, \mathrm{CDCl}_{3}\right) \delta 7.73(\mathrm{~d}$, $J=8.0 \mathrm{~Hz}, 2 \mathrm{H}), 7.30-7.36(\mathrm{~m}, 7 \mathrm{H}), 5.35(\mathrm{dd}, J=8.6,3.0 \mathrm{~Hz}, 1 \mathrm{H}), 5.15(\mathrm{~s}, 2 \mathrm{H}), 3.75(\mathrm{dd}, J=9.0$, $2.2 \mathrm{~Hz}, 1 \mathrm{H}), 3.42(\mathrm{ddd}, J=7.7,5.9,2.1 \mathrm{~Hz}, 1 \mathrm{H}), 3.09-3.17(\mathrm{~m}, 2 \mathrm{H}), 2.75(\mathrm{dd}, J=15.8,8.6 \mathrm{~Hz}, 1 \mathrm{H})$, $2.40(\mathrm{~s}, 3 \mathrm{H}), 1.84-1.91(\mathrm{~m}, 1 \mathrm{H}), 1.01(\mathrm{~d}, J=6.8 \mathrm{~Hz}, 3 \mathrm{H}), 0.92(\mathrm{~d}, J=6.8 \mathrm{~Hz}, 3 \mathrm{H}) ;{ }^{13} \mathrm{C}-\mathrm{NMR}$ $\left(100 \mathrm{MHz}, \mathrm{CDCl}_{3}\right) \delta 169.5,144.4,135.7,133.8,130.1,130.0,128.6,128.3,128.0,88.9,67.9,66.6$, 65.0, 42.0, 31.4, 21.6, 19.6, 18.5. HRMS (ESI+) calcd for $\left[\mathrm{C}_{22} \mathrm{H}_{27} \mathrm{NO}_{5} \mathrm{~S}\right]^{+}: \mathrm{m} / z$ 418.1683, found 418.1671 .

Phenyl 2-[(2S,4S)-4-isopropyl-3-tosyloxazolidin-2-yl]acetate (3d). 22\% yield; yellow oil; IR $\left(\mathrm{CH}_{2} \mathrm{Cl}_{2}\right)$ $v_{\max } 3065.2,2963.8,1758.9,1594.3,1351.3,1306.6,1164.5 \mathrm{~cm}^{-1} ;{ }^{1} \mathrm{H}-\mathrm{NMR}\left(400 \mathrm{MHz}, \mathrm{CDCl}_{3}\right) \delta 7.77$ (d, $J=8.4 \mathrm{~Hz}, 2 \mathrm{H}), 7.34-7.37(\mathrm{~m}, 4 \mathrm{H}), 7.21-7.25(\mathrm{~m}, 1 \mathrm{H}), 7.12(\mathrm{~d}, J=1.2 \mathrm{~Hz}, 2 \mathrm{H}), 5.42$ (dd, $J=8.2$, $3.4 \mathrm{~Hz}, 1 \mathrm{H}), 3.82(\mathrm{dd}, J=9.2,2.4 \mathrm{~Hz}, 1 \mathrm{H}), 3.44(\mathrm{ddd}, J=7.8,6.0,2.0 \mathrm{~Hz}, 1 \mathrm{H}), 3.34(\mathrm{dd}, J=15.8$, $3.4 \mathrm{~Hz}, 1 \mathrm{H}), 3.16(\mathrm{dd}, J=9.2,6.4 \mathrm{~Hz}, 1 \mathrm{H}), 2.93(\mathrm{dd}, J=15.8,8.2 \mathrm{~Hz}, 1 \mathrm{H}), 2.43$ (s, 3H), 1.93-1.99 (m, $1 \mathrm{H}), 1.05(\mathrm{~d}, J=6.8 \mathrm{~Hz}, 3 \mathrm{H}), 0.98(\mathrm{~d}, J=6.8 \mathrm{~Hz}, 3 \mathrm{H}) ;{ }^{13} \mathrm{C}-\mathrm{NMR}\left(100 \mathrm{MHz}, \mathrm{CDCl}_{3}\right) \delta 168.2,150.5$, $144.5,133.7,130.1$, 129.4, 128.1, 126.0, 121.6, 88.9, 67.9, 65.0, 42.2, 31.4, 21.6, 19.6, 18.5; HRMS (ESI+) calcd for $\left[\mathrm{C}_{21} \mathrm{H}_{25} \mathrm{NO}_{5} \mathrm{~S}\right]^{+}: m / z$ 404.1526, found 404.1510 .

1-[(2S,4S)-4-Isopropyl-3-tosyloxazolidin-2-yl]propan-2-one (3e). 53\% yield; light yellow oil; IR $\left(\mathrm{CH}_{2} \mathrm{Cl}_{2}\right) v_{\max } 2963.3,1719.4,1597.9,1348.5,1164.1 \mathrm{~cm}^{-1} ;{ }^{1} \mathrm{H}-\mathrm{NMR}\left(400 \mathrm{MHz}, \mathrm{CDCl}_{3}\right) \delta 7.65(\mathrm{~d}$, $J=8.4 \mathrm{~Hz}, 2 \mathrm{H}), 7.27(\mathrm{~d}, J=8.4 \mathrm{~Hz}, 2 \mathrm{H}), 5.19(\mathrm{dd}, J=8.4,2.4 \mathrm{~Hz}, 1 \mathrm{H}), 3.66(\mathrm{dd}, J=9.0,1.8 \mathrm{~Hz}, 1 \mathrm{H})$, 3.31 (ddd, $J=7.6,6.0,1.6 \mathrm{~Hz}, 1 \mathrm{H}), 3.12(\mathrm{dd}, J=17.0,2.6 \mathrm{~Hz}, 1 \mathrm{H}), 2.99$ (dd, $J=9.0,6.2 \mathrm{~Hz}, 1 \mathrm{H})$, $2.78(\mathrm{dd}, J=16.8,8.4 \mathrm{~Hz}, 1 \mathrm{H}), 2.35(\mathrm{~s}, 3 \mathrm{H}), 2.13$, (s, 3H), 1.76-1.85 (m, 1H), $0.94(\mathrm{~d}, J=6.8 \mathrm{~Hz}, 3 \mathrm{H})$, $0.86(\mathrm{~d}, J=6.8 \mathrm{~Hz}, 3 \mathrm{H}) ;{ }^{13} \mathrm{C}-\mathrm{NMR}\left(100 \mathrm{MHz}, \mathrm{CDCl}_{3}\right) \delta 205.1,144.3,133.6,130.0,127.9,88.3,67,8$, 64.7, 50.3, 31.4, 30.7, 21.5, 19.5, 18.4; HRMS (ESI+) calcd for $\left[\mathrm{C}_{16} \mathrm{H}_{23} \mathrm{NO}_{4} \mathrm{~S}\right]^{+}: \mathrm{m} / z$ 326.1421, found 326.1416 .

2-[(2S,4S)-4-Isopropyl-3-tosyloxazolidin-2-yl]-1-phenylethanone (3f). 55\% yield; yellow solid; IR $\left(\mathrm{CH}_{2} \mathrm{Cl}_{2}\right) v_{\max } 3062.5,2963.3,1686.1,1597.3,1349.4,1164.5 \mathrm{~cm}^{-1} ;{ }^{1} \mathrm{H}-\mathrm{NMR}\left(400 \mathrm{MHz}, \mathrm{CDCl}_{3}\right) \delta$ $7.95(\mathrm{~d}, J=6.8 \mathrm{~Hz}, 2 \mathrm{H}), 7.72(\mathrm{~d}, J=8.0 \mathrm{~Hz}, 2 \mathrm{H}), 7.41-7.53(\mathrm{~m}, 3 \mathrm{H}), 7.31(\mathrm{~d}, J=12.0 \mathrm{~Hz}, 2 \mathrm{H}), 5.45$ $(\mathrm{dd}, J=8.8,2.0 \mathrm{~Hz}, 1 \mathrm{H}), 3.74(\mathrm{dq}, J=12.8,2.0 \mathrm{~Hz}, 2 \mathrm{H}), 3.35-3.43(\mathrm{~m}, 2 \mathrm{H}), 3.06(\mathrm{dd}, J=8.8,6.0 \mathrm{~Hz}$, $1 \mathrm{H}), 2.37(\mathrm{~s}, 3 \mathrm{H}), 1.86-1.95(\mathrm{~m}, 1 \mathrm{H}), 1.02(\mathrm{~d}, J=7.2 \mathrm{~Hz}, 3 \mathrm{H}), 0.94(\mathrm{~d}, J=6.8 \mathrm{~Hz}, 3 \mathrm{H}) ;{ }^{13} \mathrm{C}-\mathrm{NMR}$ $\left(100 \mathrm{MHz}, \mathrm{CDCl}_{3}\right) \delta 196.6,144.4,136.6,133.6,133.5,130.1,128.7,128.2,128.0,89.0,67.9,64.8$, $45.9,31.5,21.5,19.6,18.5$; HRMS (ESI+) calcd for $\left[\mathrm{C}_{21} \mathrm{H}_{25} \mathrm{NO}_{4} \mathrm{~S}\right]^{+}: m / z 388.1577$, found 388.1568.

1-(3,4-Dichlorophenyl)-2-[(2S,4S)-4-isopropyl-3-tosyloxazolidin-2-yl]ethanone (3g). $28 \% \quad$ yield; yellow solid; IR $\left(\mathrm{CH}_{2} \mathrm{Cl}_{2}\right) v_{\max }$ 3064.1, 2963.2, 1691.3, 1584.2, 1350.0, $1164.8 \mathrm{~cm}^{-1}$; ${ }^{1} \mathrm{H}-\mathrm{NMR}$ $\left(400 \mathrm{MHz}, \mathrm{CDCl}_{3}\right) \delta 8.02(\mathrm{~d}, J=2.0 \mathrm{~Hz}, 2 \mathrm{H}), 7.78(\mathrm{dd}, J=8.4,2.0 \mathrm{~Hz}, 1 \mathrm{H}), 7.72(\mathrm{~d}, J=8.4 \mathrm{~Hz}, 2 \mathrm{H})$, 
$7.53(\mathrm{~d}, J=8.4 \mathrm{~Hz}, 1 \mathrm{H}), 7.33(\mathrm{~d}, J=8.4 \mathrm{~Hz}, 2 \mathrm{H}), 5.38(\mathrm{dd}, J=8.4,2.0 \mathrm{~Hz}, 1 \mathrm{H}), 3.76(\mathrm{dd}, J=9.2$, $1.6 \mathrm{~Hz}, 1 \mathrm{H}), 3.71$ (dd, $J=16.6,2.2 \mathrm{~Hz}, 1 \mathrm{H}), 3.39$ (ddd, $J=7.6,6.0,1.6 \mathrm{~Hz}, 1 \mathrm{H}), 3.06$ (dd, $J=9.0$, $6.2 \mathrm{~Hz}, 1 \mathrm{H}), 3.06(\mathrm{dd}, J=9.0,6.2 \mathrm{~Hz}, 1 \mathrm{H}), 2.41(\mathrm{~s}, 3 \mathrm{H}), 1.85-1.92(\mathrm{~m}, 1 \mathrm{H}), 1.02(\mathrm{~d}, J=7.2 \mathrm{~Hz}, 3 \mathrm{H})$, $0.94(\mathrm{~d}, J=6.8 \mathrm{~Hz}, 3 \mathrm{H}) ;{ }^{13} \mathrm{C}-\mathrm{NMR}\left(100 \mathrm{MHz}, \mathrm{CDCl}_{3}\right) \delta 194.5,144.5,138.0,136.1,133.5,133.4$, $130.8,130.2,130.1,128.0,127.4,88.7,67.9,64.8,46.0,31.5,21.6,19.6,18.5$; HRMS (ESI+) calcd for $\left[\mathrm{C}_{21} \mathrm{H}_{23} \mathrm{Cl}_{2} \mathrm{NO}_{4} \mathrm{~S}\right]^{+}: \mathrm{m} / z$ 456.0798, found 456.0756 .

1-(3,4-Dimethoxyphenyl)-2-[(2S,4S)-4-isopropyl-3-tosyloxazolidin-2-yl]ethanone (3h). 58\% yield; yellow oil; IR $\left(\mathrm{CH}_{2} \mathrm{Cl}_{2}\right) v_{\max } 3068.2,2962.9,1673.5,1595.7,1347.3,1164.0 \mathrm{~cm}^{-1} ;{ }^{1} \mathrm{H}-\mathrm{NMR}$ $\left(400 \mathrm{MHz} \mathrm{CDCl}_{3}\right) \delta 7.66(\mathrm{~d}, J=8.4 \mathrm{~Hz}, 2 \mathrm{H}), 7.54(\mathrm{dd}, J=8.4,2.0 \mathrm{~Hz}, 1 \mathrm{H}), 7.25(\mathrm{~d}, J=8.4 \mathrm{~Hz}, 2 \mathrm{H})$, $5.36(\mathrm{dd}, J=8.8,2.0 \mathrm{~Hz}, 1 \mathrm{H}), 3.84(\mathrm{~s}, 3 \mathrm{H}), 3.83(\mathrm{~s}, 3 \mathrm{H}), 3.61-3.70(\mathrm{~m}, 2 \mathrm{H}), 3.33$ (ddd, $J=7.5,6.1$, $1.7 \mathrm{~Hz}, 1 \mathrm{H}), 3.24$ (dd, $J=16.4,8.8 \mathrm{~Hz}, 1 \mathrm{H}), 2.32$ (s, 3H), 1.80-1.89 (m, 1H), 0.95 (d, $J=7.2 \mathrm{~Hz}, 3 \mathrm{H})$, $0.87(\mathrm{~d}, J=6.8 \mathrm{~Hz}, 3 \mathrm{H}) ;{ }^{13} \mathrm{C}-\mathrm{NMR}\left(100 \mathrm{MHz}, \mathrm{CDCl}_{3}\right) \delta 195.1,153.6,149.0,144.3,133.6,130.0$, $129.8,128.0,123.2,110.2,89.2,67.8,64.7,56.0,55.9,45.4,31.4,21.4,19.6,18.4$; HRMS (ESI+) calcd for $\left[\mathrm{C}_{23} \mathrm{H}_{29} \mathrm{NO}_{6} \mathrm{~S}\right]^{+}: \mathrm{m} / z$ 448.1788, found 448.1790 .

1-(4-Fluorophenyl)-2-[(2S,4S)-4-isopropyl-3-tosyloxazolidin-2-yl]ethanone (3i). 63\% yield; brown oil; IR $\left(\mathrm{CH}_{2} \mathrm{Cl}_{2}\right) v_{\max } 3068.8,2963.8,1686.0,1597.3,1349.6,1164.7 \mathrm{~cm}^{-1} ;{ }^{1} \mathrm{H}-\mathrm{NMR}\left(400 \mathrm{MHz}, \mathrm{CDCl}_{3}\right)$ $\delta 7.97(\mathrm{t}, J=6.8 \mathrm{~Hz}, 2 \mathrm{H}), 7.70(\mathrm{~d}, J=7.6 \mathrm{~Hz}, 2 \mathrm{H}), 7.30(\mathrm{~d}, J=8.0 \mathrm{~Hz}, 2 \mathrm{H}), 7.09(\mathrm{t}, J=8.0 \mathrm{~Hz}, 2 \mathrm{H})$, $5.40(\mathrm{dd}, J=8.6,1.8 \mathrm{~Hz}, 1 \mathrm{H}), 3.67-3.74(\mathrm{~m}, 2 \mathrm{H}), 3.29-3.40(\mathrm{~m}, 2 \mathrm{H}), 3.04(\mathrm{dd}, J=8.8,6.4 \mathrm{~Hz}, 1 \mathrm{H})$, $2.37(\mathrm{~s}, 3 \mathrm{H}), 1.84-1.92(\mathrm{~m}, 1 \mathrm{H}), 1.00(\mathrm{~d}, J=6.8 \mathrm{~Hz}, 3 \mathrm{H}), 0.92(\mathrm{~d}, J=6.8 \mathrm{~Hz}, 3 \mathrm{H}) ;{ }^{13} \mathrm{C}-\mathrm{NMR}$ $\left(100 \mathrm{MHz}, \mathrm{CDCl}_{3}\right) \delta 195.0,167.2,164.6,144.4,133.6,133.1,131.0,130.1,130.0,128.0,115.8,115.6$, 89.0, 67.9, 64.8, 45.8, 31.5, 21.5, 19.6, 18.5; HRMS (ESI+) calcd for $\left[\mathrm{C}_{21} \mathrm{H}_{24} \mathrm{FNO}_{4} \mathrm{~S}\right]^{+}: \mathrm{m} / z$ 406.1483, found 406.1477 .

2-[(2S,4S)-4-Isopropyl-3-tosyloxazolidin-2-yl]-1-(naphth-1-yl)ethanone (3j). 70\% yield; yellow oil; IR $\left(\mathrm{CH}_{2} \mathrm{Cl}_{2}\right) v_{\max } 3051.1,2963.2,1680.3,1595.9,1348.4,1164.0 \mathrm{~cm}^{-1} ;{ }^{1} \mathrm{H}-\mathrm{NMR}\left(400 \mathrm{MHz}, \mathrm{CDCl}_{3}\right)$ $\delta 8.72(\mathrm{~d}, J=8.4 \mathrm{~Hz}, 1 \mathrm{H}), 7.96-7.98(\mathrm{~m}, 2 \mathrm{H}), 7.84(\mathrm{~d}, J=8.0 \mathrm{~Hz}, 1 \mathrm{H}), 7.77(\mathrm{~d}, J=8.0 \mathrm{~Hz}, 2 \mathrm{H})$, $7.48-7.59(\mathrm{~m}, 3 \mathrm{H}), 7.27(\mathrm{~d}, J=11.6 \mathrm{~Hz}, 2 \mathrm{H}), 5.55(\mathrm{dd}, J=8.8,2.4 \mathrm{~Hz}, 1 \mathrm{H}), 3.92(\mathrm{dd}, J=16.6,2.2 \mathrm{~Hz}$, $1 \mathrm{H}), 3.79(\mathrm{dd}, J=9.0,1.8 \mathrm{~Hz}, 1 \mathrm{H}), 3.45$ (ddd, $J=13.5,6.7,4.5 \mathrm{~Hz}, 2 \mathrm{H}), 3.10$ (dd, $J=9.0,6.2 \mathrm{~Hz}$, $1 \mathrm{H}), 2.37(\mathrm{~s}, 3 \mathrm{H}), 1.90-1.98(\mathrm{~m}, 1 \mathrm{H}), 1.05(\mathrm{~d}, J=6.8 \mathrm{~Hz}, 3 \mathrm{H}), 0.96(\mathrm{~d}, J=6.4 \mathrm{~Hz}, 3 \mathrm{H}) ;{ }^{13} \mathrm{C}-\mathrm{NMR}$ $\left(100 \mathrm{MHz}, \mathrm{CDCl}_{3}\right) \delta 200.3,144.4,135.0,134.0,133.7,133.3,130.2,130.1,128.5,128.1,126.6,126.0$, 124.4, 89.4, 68.0, 64.9, 49.1, 31.6, 21.5, 19.7, 18.6; HRMS (ESI+) calcd for $\left[\mathrm{C}_{25} \mathrm{H}_{27} \mathrm{NO}_{4} \mathrm{~S}\right]^{+}: \mathrm{m} / z$ 438.1734, found 438.1730 .

2-[(2S,4S)-4-Isopropyl-3-tosyloxazolidin-2-yl]-1-(thien-2-yl)ethanone (3k). 37\% yield; brown solid; IR $\left(\mathrm{CH}_{2} \mathrm{Cl}_{2}\right) v_{\max } 3102.5,2963.1,1660.1,1597.6,1348.8,1164.0 \mathrm{~cm}^{-1} ;{ }^{1} \mathrm{H}-\mathrm{NMR}\left(400 \mathrm{MHz}, \mathrm{CDCl}_{3}\right)$ $\delta 7.77(\mathrm{~d}, J=1.2 \mathrm{~Hz}, 1 \mathrm{H}), 7.72(\mathrm{~d}, J=8.4 \mathrm{~Hz}, 2 \mathrm{H}), 7.64(\mathrm{dd}, J=5.0,1.0 \mathrm{~Hz}, 1 \mathrm{H}), 7.32(\mathrm{~d}, J=8.0 \mathrm{~Hz}$, 2H), $7.12(\mathrm{dd}, J=5.0,3.8 \mathrm{~Hz}, 1 \mathrm{H}), 5.39$ (dd, $J=8.4,2.4 \mathrm{~Hz}, 1 \mathrm{H}), 3.75(\mathrm{dd}, J=9.2,2.0 \mathrm{~Hz}, 1 \mathrm{H}), 3.64$ (dd, $J=15.8,2.2 \mathrm{~Hz}, 1 \mathrm{H}), 3.39$ (ddd, $J=7.6,6.0,1.8 \mathrm{~Hz}, 1 \mathrm{H}), 3.30$ (dd, $J=16.0,8.4 \mathrm{~Hz}, 1 \mathrm{H}), 3.06$ $(\mathrm{dd}, J=8.8,6.0 \mathrm{~Hz}, 1 \mathrm{H}), 2.40(\mathrm{~s}, 3 \mathrm{H}), 1.86-1.95(\mathrm{~m}, 1 \mathrm{H}), 1.01(\mathrm{~d}, J=6.8 \mathrm{~Hz}, 3 \mathrm{H}), 0.94(\mathrm{~d}, J=6.8 \mathrm{~Hz}$, $3 \mathrm{H}) ;{ }^{13} \mathrm{C}-\mathrm{NMR}\left(100 \mathrm{MHz}, \mathrm{CDCl}_{3}\right) \delta 189.1,144.4,144.1,134.4,133.6,132.8,130.1,128.3,128.0$, 
89.0, 67.9, 64.8, 46.5, 31.5, 21.6, 19.6, 18.5; HRMS (ESI+) calcd for $\left[\mathrm{C}_{19} \mathrm{H}_{23} \mathrm{NO}_{4} \mathrm{~S}_{2}\right]^{+}: \mathrm{m} / z$ 394.1141, found 394.1152 .

Methyl 2-[(2S,4S)-4-methyl-3-tosyloxazolidin-2-yl]acetate (4a). 68\% yield; white solid; IR $\left(\mathrm{CH}_{2} \mathrm{Cl}_{2}\right)$ $v_{\max }$ 2983.0, 1739.9, 1598.2, 1350.8, $1164.8 \mathrm{~cm}^{-1} ;{ }^{1} \mathrm{H}-\mathrm{NMR}\left(400 \mathrm{MHz}, \mathrm{CDCl}_{3}\right) \delta 7.69(\mathrm{~d}, J=1.6 \mathrm{~Hz}$, 2H), $7.30(\mathrm{~d}, J=8.0 \mathrm{~Hz}, 2 \mathrm{H}), 5.29(\mathrm{dd}, J=8.8,2.8 \mathrm{~Hz}, 1 \mathrm{H}), 3.65-3.70(\mathrm{~m}, 4 \mathrm{H}), 3.52(\mathrm{dd}, J=9.0$, $4.2 \mathrm{~Hz}, 1 \mathrm{H}), 3.41(\mathrm{dd}, J=8.8,6.0 \mathrm{~Hz}, 1 \mathrm{H}), 3.04(\mathrm{dd}, J=16.0,3.2 \mathrm{~Hz}, 1 \mathrm{H}), 2.69$ (dd, $J=15.6,8.8 \mathrm{~Hz}$, 1H), $2.38(\mathrm{~s}, 3 \mathrm{H}), 1.29$ (d, $J=6.4 \mathrm{~Hz}, 3 \mathrm{H}) ;{ }^{13} \mathrm{C}-\mathrm{NMR}\left(100 \mathrm{MHz}, \mathrm{CDCl}_{3}\right) \delta 169.9,144.4,133.6,130.0$, $127.8,127.1,88.9,71.8,55.1,51.9,51.8,42.1,21.5,20.9$; HRMS (ESI+) calcd for $\left[\mathrm{C}_{14} \mathrm{H}_{19} \mathrm{NO}_{5} \mathrm{~S}\right]^{+}$: $\mathrm{m} / \mathrm{z} 314.1057$, found 314.1050.

Ethyl 2-[(2S,4S)-4-methyl-3-tosyloxazolidin-2-yl]acetate (4b). $68 \%$ yield; white solid; IR $\left(\mathrm{CH}_{2} \mathrm{Cl}_{2}\right)$ $v_{\max }$ 2982.7, 1736.4, 1598.3, 1351.1, $1164.9 \mathrm{~cm}^{-1} ;{ }^{1} \mathrm{H}-\mathrm{NMR}\left(400 \mathrm{MHz}, \mathrm{CDCl}_{3}\right) \delta 7.65(\mathrm{~d}, J=8.4 \mathrm{~Hz}$, 2H), $7.26(\mathrm{~d}, J=8.0 \mathrm{~Hz}, 2 \mathrm{H}), 5.25(\mathrm{dd}, J=8.6,3.0 \mathrm{~Hz}, 1 \mathrm{H}), 4.08(\mathrm{q}, J=7.1 \mathrm{~Hz}, 2 \mathrm{H}), 3.65$ (ddd, $J=8.3,4.3,2.1 \mathrm{~Hz}, 1 \mathrm{H}), 3.48(\mathrm{dd}, J=9.0,4.2 \mathrm{~Hz}, 1 \mathrm{H}), 3.37(\mathrm{dd}, J=8.8,6.0 \mathrm{~Hz}, 1 \mathrm{H}), 2.98(\mathrm{dd}$, $J=15.8,3.0 \mathrm{~Hz}, 1 \mathrm{H}), 2.63(\mathrm{dd}, J=15.6 .8 .8 \mathrm{~Hz}, 1 \mathrm{H}), 2.33(\mathrm{~s}, 3 \mathrm{H}), 1.24(\mathrm{~d}, J=6.4 \mathrm{~Hz}, 3 \mathrm{H}), 1.18$ (t, $J=7.2 \mathrm{~Hz}, 3 \mathrm{H}) ;{ }^{13} \mathrm{C}-\mathrm{NMR}\left(100 \mathrm{MHz}, \mathrm{CDCl}_{3}\right) \delta 169.4,144.3,133.6,130.0,127.8,127.1,88.9,71.8$, 60.7, 55.0, 42.2, 21.4, 20.8, 14.1; HRMS (ESI+) calcd for $\left[\mathrm{C}_{15} \mathrm{H}_{21} \mathrm{NO}_{5} \mathrm{~S}\right]^{+}: \mathrm{m} / z$ 328.1213, found 328.1207 .

Benzyl 2-[(2S,4S)-4-methyl-3-tosyloxazolidin-2-yl]acetate (4c). $64 \%$ yield; yellow oil; IR $\left(\mathrm{CH}_{2} \mathrm{Cl}_{2}\right)$ $v_{\max }$ 3064.8, 2979.9, 1738.1, 1598.1, 1351.1, $1165.1 \mathrm{~cm}^{-1} ;{ }^{1} \mathrm{H}-\mathrm{NMR}\left(400 \mathrm{MHz}, \mathrm{CDCl}_{3}\right) \delta 7.73(\mathrm{~d}$, $J=8.4 \mathrm{~Hz}, 2 \mathrm{H}), 7.28-7.36(\mathrm{~m}, 6 \mathrm{H}), 5.38(\mathrm{dd}, J=8.6,3.0 \mathrm{~Hz}, 1 \mathrm{H}), 5.16(\mathrm{~s}, 2 \mathrm{H}), 3.73$ (ddd, $J=8.5,4.1$, $2.1 \mathrm{~Hz}, 1 \mathrm{H}), 3.55(\mathrm{dd}, J=8.8,4.0 \mathrm{~Hz}, 1 \mathrm{H}), 3.46(\mathrm{dd}, J=9.0,6.2 \mathrm{~Hz}, 1 \mathrm{H}), 3.14(\mathrm{dd}, J=15.8,3.0 \mathrm{~Hz}$, 1H), 2.79 (dd, $J=15.8,8.6 \mathrm{~Hz}, 1 \mathrm{H}), 2.41(\mathrm{~s}, 3 \mathrm{H}), 1.32$ (d, $J=6.4 \mathrm{~Hz}, 3 \mathrm{H}) ;{ }^{13} \mathrm{C}-\mathrm{NMR}(100 \mathrm{MHz}$, $\left.\mathrm{CDCl}_{3}\right) \delta 169.4,144.4,135.7,133.6,130.1,130.0,128.6,128.5,128.3,127.9,127.2,89.0,71.9,66.6$, 55.2, 42.3, 21.6, 20.9; HRMS (ESI+) calcd for $\left[\mathrm{C}_{20} \mathrm{H}_{23} \mathrm{NO}_{5} \mathrm{~S}\right]^{+}: \mathrm{m} / z$ 390.1317, found 390.1346.

Phenyl 2-[(2S,4S)-4-methyl-3-tosyloxazolidin-2-yl]acetate (4d). 14\% yield; yellow oil; $\mathrm{IR}\left(\mathrm{CH}_{2} \mathrm{Cl}_{2}\right)$ $v_{\max }$ 3068.2, 2980.3, 1758.4, 1593.7, 1350.8, $1164.3 \mathrm{~cm}^{-1} ;{ }^{1} \mathrm{H}-\mathrm{NMR}\left(400 \mathrm{MHz}, \mathrm{CDCl}_{3}\right) \delta 7.77(\mathrm{~d}$, $J=8.4 \mathrm{~Hz}, 2 \mathrm{H}), 7.34-7.40(\mathrm{~m}, 5 \mathrm{H}), 7.23-7.25(\mathrm{~m}, 1 \mathrm{H}), 7.12(\mathrm{~d}, J=1.2 \mathrm{~Hz}, 2 \mathrm{H}), 5.45(\mathrm{dd}, J=8.2$, $3.4 \mathrm{~Hz}, 1 \mathrm{H}), 3.76(\mathrm{ddd}, J=8.4,4.4,2.0 \mathrm{~Hz}, 1 \mathrm{H}), 3.64(\mathrm{dd}, J=8.8,4.0 \mathrm{~Hz}, 1 \mathrm{H}), 3.52(\mathrm{dd}, J=8.8$, $6.4 \mathrm{~Hz}, 1 \mathrm{H}), 3.34$ (dd, $J=15.8,3.4 \mathrm{~Hz}, 1 \mathrm{H}), 2.99$ (dd, $J=15.8,8.2 \mathrm{~Hz}, 1 \mathrm{H}), 2.44$ (s, 3H), 1.39 (d, $J=6.4 \mathrm{~Hz}, 3 \mathrm{H}) ;{ }^{13} \mathrm{C}-\mathrm{NMR}\left(100 \mathrm{MHz}, \mathrm{CDCl}_{3}\right) \delta 168.0,150.5,144.4,133.6,130.1,129.4,129.1,127.9$, 126.0, 121.6, 88.9, 72.0, 55.2, 42.5, 21.6, 21.0; HRMS (ESI+) calcd for $\left[\mathrm{C}_{19} \mathrm{H}_{21} \mathrm{NO}_{5} \mathrm{~S}\right]^{+}: \mathrm{m} / z$ 376.1213, found 376.1204 .

1-[(2S,4S)-4-Methyl-3-tosyloxazolidin-2-yl]propan-2-one (4e). $68 \%$ yield; yellow oil; $\mathrm{IR}\left(\mathrm{CH}_{2} \mathrm{Cl}_{2}\right) v_{\max }$ 2980.2, 1715.8, 1598.2, 1348.8, $1164.6 \mathrm{~cm}^{-1}$; ${ }^{1} \mathrm{H}-\mathrm{NMR}\left(400 \mathrm{MHz}, \mathrm{CDCl}_{3}\right) \delta 7.66(\mathrm{~d}, J=8.4 \mathrm{~Hz}, 2 \mathrm{H})$, $7.28(\mathrm{~d}, J=8.0 \mathrm{~Hz}, 2 \mathrm{H}), 5.23(\mathrm{dd}, J=8.4,2.4 \mathrm{~Hz}, 1 \mathrm{H}), 3.65$ (ddd, $J=7.8,4.6,1.8 \mathrm{~Hz}, 1 \mathrm{H}), 3.47$ (dd, $J=8.8,3.6 \mathrm{~Hz}, 1 \mathrm{H}), 3.32(\mathrm{dd}, J=9.0,6.2 \mathrm{~Hz}, 1 \mathrm{H}), 3.11(\mathrm{dd}, J=16.6,2.6 \mathrm{~Hz}, 1 \mathrm{H}), 2.84(\mathrm{dd}, J=16.4$. $8.4 \mathrm{~Hz}, 1 \mathrm{H}), 2.36(\mathrm{~s}, 3 \mathrm{H}), 2.14(\mathrm{~s}, 3 \mathrm{H}), 1.26(\mathrm{~d}, J=6.8 \mathrm{~Hz}, 3 \mathrm{H}) ;{ }^{13} \mathrm{C}-\mathrm{NMR}\left(100 \mathrm{MHz}, \mathrm{CDCl}_{3}\right) \delta 205.1$, 
144.3, 133.5, 130.0, 127.8, 126.9, 88.4, 71.8, 54.9, 50.6, 30.8, 21.5, 21.0; HRMS (ESI+) calcd for $\left[\mathrm{C}_{14} \mathrm{H}_{19} \mathrm{NO}_{4} \mathrm{~S}\right]^{+}: m / z 298.1108$, found 298.1128 .

2-[(2S,4S)-4-Methyl-3-tosyloxazolidin-2-yl]-1-phenylethanone (4f). 57\% yield; white solid; IR $\left(\mathrm{CH}_{2} \mathrm{Cl}_{2}\right) v_{\max } 3062.3,2980.2,1686.7,1597.3,1348.9,1164.5 \mathrm{~cm}^{-1} ;{ }^{1} \mathrm{H}-\mathrm{NMR}\left(400 \mathrm{MHz}, \mathrm{CDCl}_{3}\right)$ $\delta 7.97(\mathrm{~d}, J=7.2 \mathrm{~Hz}, 2 \mathrm{H}), 7.73(\mathrm{~d}, J=8.0 \mathrm{~Hz}, 2 \mathrm{H}), 7.53-7.74(\mathrm{~m}, 1 \mathrm{H}), 7.45(\mathrm{t}, J=7.6 \mathrm{~Hz}, 2 \mathrm{H}), 7.31$ $(\mathrm{d}, J=8.0 \mathrm{~Hz}, 2 \mathrm{H}), 5.50(\mathrm{dd}, J=8.8,2.0 \mathrm{~Hz}, 1 \mathrm{H}), 3.71-3.75(\mathrm{~m}, 2 \mathrm{H}), 3.55(\mathrm{dd}, J=8.8,3.6 \mathrm{~Hz}, 1 \mathrm{H})$, $3.37-3.45(\mathrm{~m}, 2 \mathrm{H}), 2.39(\mathrm{~s}, 3 \mathrm{H}), 1.35(\mathrm{~d}, J=6.4 \mathrm{~Hz}, 3 \mathrm{H}) ;{ }^{13} \mathrm{C}-\mathrm{NMR}\left(100 \mathrm{MHz}, \mathrm{CDCl}_{3}\right) \delta 196.6,144.4$, 136.6, 133.5, 130.1, 130.0, 129.6, 128.7, 128.3, 127.9, 89.0, 71.9, 55.0, 46.3, 21.6, 21.1; HRMS (ESI+) calcd for $\left[\mathrm{C}_{19} \mathrm{H}_{21} \mathrm{NO}_{4} \mathrm{~S}\right]^{+}: m / z 360.1264$, found 360.1260 .

1-(3,4-Dichlorophenyl)-2-[(2S,4S)-4-methyl-3-tosyloxazolidin-2-yl]ethanone (4g). 41\% yield; brown oil; IR $\left(\mathrm{CH}_{2} \mathrm{Cl}_{2}\right) v_{\max } 3065.8,2980.5,1692.7,1584.3,1349.3,1164.8 \mathrm{~cm}^{-1}$; ${ }^{1} \mathrm{H}-\mathrm{NMR}(400 \mathrm{MHz}$, $\left.\mathrm{CDCl}_{3}\right) \delta 8.03(\mathrm{~d}, J=2.0 \mathrm{~Hz}, 1 \mathrm{H}), 7.79(\mathrm{dd}, J=8.4,2.0 \mathrm{~Hz}, 1 \mathrm{H}), 7.71(\mathrm{~d}, J=8.4 \mathrm{~Hz}, 2 \mathrm{H}), 7.32(\mathrm{~d}$, $J=8.0 \mathrm{~Hz}, 2 \mathrm{H}), 5.42(\mathrm{dd}, J=8.4,2.0 \mathrm{~Hz}, 1 \mathrm{H}), 3.67-3.74(\mathrm{~m}, 2 \mathrm{H}), 3.55(\mathrm{dd}, J=9.0,3.4 \mathrm{~Hz}, 1 \mathrm{H})$, $3.32-3.40(\mathrm{~m}, 2 \mathrm{H}), 2.40(\mathrm{~s}, 3 \mathrm{H}), 1.34(\mathrm{~d}, J=6.4 \mathrm{~Hz}, 3 \mathrm{H}) ;{ }^{13} \mathrm{C}-\mathrm{NMR}\left(100 \mathrm{MHz}, \mathrm{CDCl}_{3}\right) \delta 194.4,144.5$, 138.4, 137.0, 134.0, 133.4, 131.1, 130.8, 130.0, 128.3, 127.4, 88.8, 72.0, 55.0, 46.3, 21.6, 21.1; HRMS $(\mathrm{ESI}+)$ calcd for $\left[\mathrm{C}_{19} \mathrm{H}_{19} \mathrm{Cl}_{2} \mathrm{NO}_{4} \mathrm{~S}\right]^{+}: \mathrm{m} / z$ 428.0485, found 428.0476 .

1-(3,4-Dimethoxyphenyl)-2-[(2S,4S)-4-methyl-3-tosyloxazolidin-2-yl]ethanone (4h). 90\% yield; yellow oil; IR $\left(\mathrm{CH}_{2} \mathrm{Cl}_{2}\right) v_{\max } 3064.1,2974.2,1672.7,1595.4,1346.4,1163.4 \mathrm{~cm}^{-1} ;{ }^{1} \mathrm{H}-\mathrm{NMR}(400 \mathrm{MHz}$, $\left.\mathrm{CDCl}_{3}\right) \delta 7.64(\mathrm{~d}, J=8.0 \mathrm{~Hz}, 2 \mathrm{H}), 7.53(\mathrm{dd}, J=8.4,2.0 \mathrm{~Hz}, 1 \mathrm{H}), 7.43(\mathrm{~d}, J=2.0 \mathrm{~Hz}, 1 \mathrm{H}), 7.22(\mathrm{~d}$, $J=8.0 \mathrm{~Hz}, 2 \mathrm{H}), 6.81(\mathrm{~d}, J=8.4 \mathrm{~Hz}, 1 \mathrm{H}), 5.40(\mathrm{dd}, J=8.8,2.0 \mathrm{~Hz}, 1 \mathrm{H}), 3.78-3.81(\mathrm{~m}, 7 \mathrm{H}), 3.62$ (ddd, $J=12.5,6.1,3.3 \mathrm{~Hz}, 1 \mathrm{H}), 3.56(\mathrm{~d}, J=2.0 \mathrm{~Hz}, 1 \mathrm{H}), 3.47(\mathrm{dd}, J=8.8,3.6 \mathrm{~Hz}, 1 \mathrm{H}), 3.26-3.32(\mathrm{~m}, 2 \mathrm{H})$, $2.29(\mathrm{~s}, 3 \mathrm{H}), 1.25(\mathrm{~d}, J=6.4 \mathrm{~Hz}, 3 \mathrm{H}) ;{ }^{13} \mathrm{C}-\mathrm{NMR}\left(100 \mathrm{MHz}, \mathrm{CDCl}_{3}\right) \delta 195.0,153.6,149.0,144.3$, $133.4,130.0,127.8,110.2,89.3,71.8,56.0,55.0,45.8,21.4,20.9$; HRMS (ESI+) calcd for $\left[\mathrm{C}_{21} \mathrm{H}_{25} \mathrm{NO}_{6} \mathrm{~S}\right]^{+}: \mathrm{m} / z$ 420.1475, found 420.1464 .

1-(4-Fluorophenyl)-2-[(2S,4S)-4-Methyl-3-tosyloxazolidin-2-yl]ethanone (4i). 51\% yield; white solid; IR $\left(\mathrm{CH}_{2} \mathrm{Cl}_{2}\right) v_{\max } 3069.4,2981.0,1686.4,1597.3,1349.2,1164.7 \mathrm{~cm}^{-1} ;{ }^{1} \mathrm{H}-\mathrm{NMR}\left(400 \mathrm{MHz}, \mathrm{CDCl}_{3}\right)$ $\delta 8.00(\mathrm{q}, J=1.1 \mathrm{~Hz}, 2 \mathrm{H}), 7.72(\mathrm{~d}, J=8.4 \mathrm{~Hz}, 2 \mathrm{H}), 7.31(\mathrm{~d}, J=8.0 \mathrm{~Hz}, 2 \mathrm{H}), 7.12(\mathrm{t}, J=8.8 \mathrm{~Hz}, 2 \mathrm{H})$, $5.46(\mathrm{dd}, J=8.8,2.0 \mathrm{~Hz}, 1 \mathrm{H}), 3.67-3.74(\mathrm{~m}, 2 \mathrm{H}), 3.55(\mathrm{dd}, J=8.8,3.6 \mathrm{~Hz}, 1 \mathrm{H}), 3.35-3.41(\mathrm{~m}, 2 \mathrm{H})$, $2.39(\mathrm{~s}, 3 \mathrm{H}), 1.34(\mathrm{~d}, J=6.4 \mathrm{~Hz}, 3 \mathrm{H}) ;{ }^{13} \mathrm{C}-\mathrm{NMR}\left(100 \mathrm{MHz}, \mathrm{CDCl}_{3}\right) \delta 195.0,167.2,164.7,144.4$, 133.4, 133.1, 131.0, 130.1, 127.9, 115.9, 89.0, 71.9, 55.0, 46.2, 21.5, 21.1; HRMS (ESI+) calcd for $\left[\mathrm{C}_{19} \mathrm{H}_{20} \mathrm{FNO}_{4} \mathrm{~S}\right]^{+}: m / z$ 378.1170, found 378.1170.

2-[(2S,4S)-4-Methyl-3-tosyloxazolidin-2-yl]-1-(naphth-1-yl)ethanone (4j). 84\% yield; brown oil; IR $\left(\mathrm{CH}_{2} \mathrm{Cl}_{2}\right) v_{\max } 3051.4,2980.3,1678.6,1596.0,1348.9,1164.0 \mathrm{~cm}^{-1} ;{ }^{1} \mathrm{H}-\mathrm{NMR}\left(400 \mathrm{MHz}, \mathrm{CDCl}_{3}\right)$ $\delta 8.73(\mathrm{~d}, J=8.4 \mathrm{~Hz}, 1 \mathrm{H}), 7.97(\mathrm{~d}, J=7.6 \mathrm{~Hz}, 2 \mathrm{H}), 7.84(\mathrm{~d}, J=8.0 \mathrm{~Hz}, 1 \mathrm{H}), 7.75(\mathrm{~d}, J=8.0 \mathrm{~Hz}, 2 \mathrm{H})$, $7.47-7.59(\mathrm{~m}, 3 \mathrm{H}), 7.28(\mathrm{~d}, J=8.0 \mathrm{~Hz}, 2 \mathrm{H}), 5.59(\mathrm{dd}, J=8.6,2.2 \mathrm{~Hz}, 1 \mathrm{H}), 3.89$ (dd, $J=16.4,2.4 \mathrm{~Hz}$, $1 \mathrm{H}), 3.75(\mathrm{ddd}, J=8.1,4.5,1.9 \mathrm{~Hz}, 1 \mathrm{H}), 3.56(\mathrm{dd}, J=9.0,3.4 \mathrm{~Hz}, 1 \mathrm{H}), 3.50(\mathrm{dd}, J=16.8,8.8 \mathrm{~Hz}$, $1 \mathrm{H}), 3.39(\mathrm{dd}, J=9.0,6.2 \mathrm{~Hz}, 1 \mathrm{H}), 2.35(\mathrm{~s}, 3 \mathrm{H}), 1.36(\mathrm{~d}, J=6.8 \mathrm{~Hz}, 3 \mathrm{H}) ;{ }^{13} \mathrm{C}-\mathrm{NMR}(100 \mathrm{MHz}$, 
$\left.\mathrm{CDCl}_{3}\right) \delta 200.2,144.4,134.9,134.0,133.5,133.3,130.2,130.0,128.6,128.1,126.6,126.0,124.5$, 89.4, 71.9, 55.1, 49.4, 21.5, 21.1; HRMS (ESI+) calcd for $\left[\mathrm{C}_{23} \mathrm{H}_{23} \mathrm{NO}_{4} \mathrm{~S}\right]^{+}: \mathrm{m} / z$ 410.1421, found 410.1421 .

2-[(2S,4S)-4-Methyl-3-tosyloxazolidin-2-yl]-1-(thien-2-yl)ethanone (4k). 49\% yield; brown solid; IR $\left(\mathrm{CH}_{2} \mathrm{Cl}_{2}\right) v_{\max } 3102.9,2980.4,1658.8,1597.7,1348.9,1164.0 \mathrm{~cm}^{-1} ;{ }^{1} \mathrm{H}-\mathrm{NMR}\left(400 \mathrm{MHz}, \mathrm{CDCl}_{3}\right)$ $\delta 7.78(\mathrm{dd}, J=3.8,1.0 \mathrm{~Hz}, 1 \mathrm{H}), 7.72(\mathrm{~d}, J=8.0 \mathrm{~Hz}, 2 \mathrm{H}), 7.65(\mathrm{dd}, J=5.0,1.0 \mathrm{~Hz}, 1 \mathrm{H}), 7.32(\mathrm{~d}$, $J=8.0 \mathrm{~Hz}, 2 \mathrm{H}), 7.13(\mathrm{dd}, J=4.8,4.0 \mathrm{~Hz}, 1 \mathrm{H}), 5.45(\mathrm{dd}, J=7.6,3.2 \mathrm{~Hz}, 1 \mathrm{H}), 3.72$ (ddd, $J=8.0,4.4$, $1.8 \mathrm{~Hz}, 1 \mathrm{H}), 3.63(\mathrm{dd}, J=16.0,2.0 \mathrm{~Hz}, 1 \mathrm{H}), 3.56(\mathrm{dd}, J=8.8,3.6 \mathrm{~Hz}, 1 \mathrm{H}), 3.32-3.41(\mathrm{~m}, 2 \mathrm{H}), 2.40$ $(\mathrm{s}, 3 \mathrm{H}), 1.34(\mathrm{~d}, J=6.4 \mathrm{~Hz}, 3 \mathrm{H}) ;{ }^{13} \mathrm{C}-\mathrm{NMR}\left(100 \mathrm{MHz}, \mathrm{CDCl}_{3}\right) \delta 189.1,144.4,144.0,134.4,133.4$, 132.9, 130.0, 128.4, 127.9, 89.0, 71.9, 55.0, 46.9, 21.6, 21.0; HRMS (ESI+) calcd for $\left[\mathrm{C}_{17} \mathrm{H}_{19} \mathrm{NO}_{4} \mathrm{~S}_{2}\right]^{+}$: $\mathrm{m} / \mathrm{z} 366.0828$, found 366.0822 .

Methyl 2-[(2S,4R)-4-benzyl-3-tosyloxazolidin-2-yl]acetate (5a). $66 \%$ yield; white solid; $\mathrm{IR}\left(\mathrm{CH}_{2} \mathrm{Cl}_{2}\right)$ $v_{\max }$ 3028.3, 2952.2, 1740.7, 1597.9, 1351.7, $1163.9 \mathrm{~cm}^{-1} ;{ }^{1} \mathrm{H}-\mathrm{NMR}\left(400 \mathrm{MHz}, \mathrm{CDCl}_{3}\right) \delta 7.77(\mathrm{~d}$, $J=8.4 \mathrm{~Hz}, 2 \mathrm{H}), 7.18-7.34(\mathrm{~m}, 7 \mathrm{H}), 5.33$ (dd, $J=8.8,2.8 \mathrm{~Hz}, 1 \mathrm{H}), 3.83$ (ddd, $J=12.8,6.4,3.2 \mathrm{~Hz}$, $1 \mathrm{H}), 3.72(\mathrm{dd}, J=9.2,3.6 \mathrm{~Hz}, 1 \mathrm{H}), 3.69(\mathrm{~s}, 3 \mathrm{H}), 3.22(\mathrm{dd}, J=9.2,6.4 \mathrm{~Hz}, 1 \mathrm{H}), 3.13(\mathrm{dd}, J=13.6$, $3.6 \mathrm{~Hz}, 1 \mathrm{H}), 3.06(\mathrm{dd}, J=15.8,3.0 \mathrm{~Hz}, 1 \mathrm{H}), 2.84(\mathrm{dd}, J=13.2,10.0 \mathrm{~Hz}, 1 \mathrm{H}), 2.60(\mathrm{dd}, J=15.8$, $8.6 \mathrm{~Hz}, 1 \mathrm{H}), 2.40(\mathrm{~s}, 3 \mathrm{H}) ;{ }^{13} \mathrm{C}-\mathrm{NMR}\left(100 \mathrm{MHz}, \mathrm{CDCl}_{3}\right) \delta 169.9,144.5,137.0,133.6,130.1,129.6$, 128.7, 127.9, 126.9, 89.0, 69.1, 60.5, 51.9, 41.8, 40.9, 21.6; HRMS (ESI+) calcd for $\left[\mathrm{C}_{20} \mathrm{H}_{23} \mathrm{NO}_{5} \mathrm{~S}\right]^{+}$: $\mathrm{m} / \mathrm{z} 390.1370$, found 390.1364 .

Ethyl 2-[(2R,4S)-4-benzyl-3-tosyloxazolidin-2-yl]acetate (5b). 81\% yield; white solid; IR $\left(\mathrm{CH}_{2} \mathrm{Cl}_{2}\right)$ $v_{\max }$ 3028.3, 2983.2, 1736.0, 1597.9, 1352.0, $1164.1 \mathrm{~cm}^{-1} ;{ }^{1} \mathrm{H}-\mathrm{NMR}\left(400 \mathrm{MHz}, \mathrm{CDCl}_{3}\right) \delta 7.75(\mathrm{dd}$, $J=8.4 \mathrm{~Hz}, 2 \mathrm{H}), 7.30(\mathrm{~d}, J=8.4 \mathrm{~Hz}, 2 \mathrm{H}), 7.26(\mathrm{~d}, J=7.6 \mathrm{~Hz}, 2 \mathrm{H}), 7.18(\mathrm{~d}, J=8.0 \mathrm{~Hz}, 3 \mathrm{H}), 5.33(\mathrm{dd}$, $J=8.8,2.8 \mathrm{~Hz}, 1 \mathrm{H}), 4.14(\mathrm{q}, J=7.2 \mathrm{~Hz}, 2 \mathrm{H}), 3.82(\mathrm{ddd}, J=13.0,6.6,3.2 \mathrm{~Hz}, 1 \mathrm{H}), 3.70(\mathrm{dd}, J=9.2$, $3.2 \mathrm{~Hz}, 1 \mathrm{H}), 3.20(\mathrm{dd}, J=9.2,6.0 \mathrm{~Hz}, 1 \mathrm{H}), 3.13(\mathrm{dd}, J=13.4,3.8 \mathrm{~Hz}, 1 \mathrm{H}), 3.05(\mathrm{dd}, J=15.8,3.0 \mathrm{~Hz}$, $1 \mathrm{H}), 2.83(\mathrm{dd}, J=13.4,9.8 \mathrm{~Hz}, 1 \mathrm{H}), 2.37(\mathrm{~s}, 3 \mathrm{H}), 1.24(\mathrm{t}, J=7.2 \mathrm{~Hz}, 3 \mathrm{H}) ;{ }^{13} \mathrm{C}-\mathrm{NMR}(100 \mathrm{MHz}$, $\left.\mathrm{CDCl}_{3}\right) \delta 169.5,144.5,137.1,133.6,130.1,129.6,128.7,127.9,126.9,89.1,69.1,60.7,60.4,42.0$, 41.0, 21.5, 14.2; HRMS (ESI+) calcd for $\left[\mathrm{C}_{21} \mathrm{H}_{25} \mathrm{NO}_{5} \mathrm{~S}\right]^{+}: \mathrm{m} / z$ 404.1526, found 404.1528.

Benzyl 2-[(2S,4R)-4-benzyl-3-tosyloxazolidin-2-yl]acetate (5c). 76\% yield; yellow oil; IR $\left(\mathrm{CH}_{2} \mathrm{Cl}_{2}\right)$ $v_{\max } 3063.3,3030.3,2948.4,1737.7,1597.8,1352.0,1164.0 \mathrm{~cm}^{-1} ;{ }^{1} \mathrm{H}-\mathrm{NMR}\left(400 \mathrm{MHz}, \mathrm{CDCl}_{3}\right) \delta 7.81$ $(\mathrm{d}, J=8.0 \mathrm{~Hz}, 2 \mathrm{H}), 7.23-7.42(\mathrm{~m}, 12 \mathrm{H}), 5.44(\mathrm{dd}, J=8.4,3.2 \mathrm{~Hz}, 1 \mathrm{H}), 5.20(\mathrm{~s}, 2 \mathrm{H}), 3.89$ (ddd, $J=12.9,6.5,3.1 \mathrm{~Hz}, 1 \mathrm{H}), 3.78(\mathrm{dd}, J=8.4,5.2 \mathrm{~Hz}, 1 \mathrm{H}), 3.27(\mathrm{dd}, J=9.2,6.4 \mathrm{~Hz}, 1 \mathrm{H}), 3.16-3.21$ $(\mathrm{m}, 2 \mathrm{H}), 2.88(\mathrm{dd}, J=13.6,10.0 \mathrm{~Hz}, 1 \mathrm{H}), 2.73(\mathrm{dd}, J=16.0,8.4 \mathrm{~Hz}, 1 \mathrm{H}), 2.43(\mathrm{~s}, 3 \mathrm{H}) ;{ }^{13} \mathrm{C}-\mathrm{NMR}$ $\left(100 \mathrm{MHz}, \mathrm{CDCl}_{3}\right) \delta 169.4,144.6,137.1,135.8,133.7,130.2,130.1,129.6,128.8,128.7,128.5,128.3$, 128.0, 127.0, 89.2, 69.2, 66.7, 60.6, 42.1, 41.0, 21.6; HRMS (ESI+) calcd for $\left[\mathrm{C}_{26} \mathrm{H}_{27} \mathrm{NO}_{5} \mathrm{~S}\right]^{+}: \mathrm{m} / \mathrm{z}$ 466.1683 , found 466.1687 .

Phenyl 2-[(2S,4R)-4-benzyl-3-tosyloxazolidin-2-yl]acetate (5d). 5\% yield; yellow oil; IR $\left(\mathrm{CH}_{2} \mathrm{Cl}_{2}\right) v_{\max }$ 3028.5, 2924.3, 1712.3, 1589.8, 1325.3, $1362.2 \mathrm{~cm}^{-1}$; ${ }^{1} \mathrm{H}-\mathrm{NMR}\left(400 \mathrm{MHz}, \mathrm{CDCl}_{3}\right) \delta 7.80(\mathrm{~d}$, 
$J=8.4 \mathrm{~Hz}, 2 \mathrm{H}), 7.05-7.42(\mathrm{~m}, 12 \mathrm{H}), 5.42(\mathrm{dd}, J=8.0,3.2 \mathrm{~Hz}, 1 \mathrm{H}), 3.86(\mathrm{ddd}, J=13.1,6.3,3.3 \mathrm{~Hz}$, $1 \mathrm{H}), 3.79(\mathrm{dd}, J=9.2,3.2 \mathrm{~Hz}, 1 \mathrm{H}), 3.33(\mathrm{dd}, J=16.0,3.2 \mathrm{~Hz}, 1 \mathrm{H}), 3.27$ (dd, $J=9.0,6.2 \mathrm{~Hz}, 1 \mathrm{H}), 3.20$ $(\mathrm{dd}, J=13.6,3.6 \mathrm{~Hz}, 1 \mathrm{H}), 2.82-2.92(\mathrm{~m}, 3 \mathrm{H}), 2.43(\mathrm{~s}, 3 \mathrm{H}) ;{ }^{13} \mathrm{C}-\mathrm{NMR}\left(100 \mathrm{MHz}, \mathrm{CDCl}_{3}\right) \delta 167.0$, $159.8,155.7,143.4,137.5,136.3,129.7,127.0,128.0,101.1,64.5,54.1,38.8,21.5$; HRMS (ESI+) calcd for $\left[\mathrm{C}_{25} \mathrm{H}_{25} \mathrm{NO}_{5} \mathrm{~S}\right]^{+}: m / z$ 452.1526, found 452.1515 .

1-[(2S,4S)-4-Benzyl-3-tosyloxazolidin-2-yl]propan-2-one (5e). 45\% yield; yellow oil; IR $\left(\mathrm{CH}_{2} \mathrm{Cl}_{2}\right) v_{\max }$ 3062.3, 2924.8, 1716.7, 1598.0, 1349.8, $1163.6 \mathrm{~cm}^{-1}$; ${ }^{1} \mathrm{H}-\mathrm{NMR}\left(400 \mathrm{MHz}, \mathrm{CDCl}_{3}\right) \delta 7.74(\mathrm{~d}$, $J=8.4 \mathrm{~Hz}, 2 \mathrm{H}), 7.32(\mathrm{~d}, J=8.0 \mathrm{~Hz}, 2 \mathrm{H}), 7.29(\mathrm{~d}, J=7.6 \mathrm{~Hz}, 2 \mathrm{H}), 7.19-7.22(\mathrm{~m}, 3 \mathrm{H}), 5.27(\mathrm{dd}$, $J=8.4,2.4 \mathrm{~Hz}, 1 \mathrm{H}), 3.80(\mathrm{ddd}, J=9.4,6.2,3.2 \mathrm{~Hz}, 1 \mathrm{H}), 3.69(\mathrm{dd}, J=9.2,2.8 \mathrm{~Hz}, 1 \mathrm{H}), 3.10-3.17$ (m, $3 \mathrm{H}), 2.83(\mathrm{dd}, J=13.6,10.0 \mathrm{~Hz}, 1 \mathrm{H}), 2.72(\mathrm{dd}, J=16.8,8.4 \mathrm{~Hz}, 1 \mathrm{H}), 2.40(\mathrm{~s}, 3 \mathrm{H}), 2.13(\mathrm{~s}, 3 \mathrm{H})$; ${ }^{13} \mathrm{C}-\mathrm{NMR}\left(100 \mathrm{MHz}, \mathrm{CDCl}_{3}\right) \delta 205.1,144.5,137.1,133.5,13071,129.7,128.7,127.9,126.9,88.6,69.0$, 60.3, 50.2, 41.0, 30.8, 21.6; HRMS (ESI+) calcd for $\left[\mathrm{C}_{20} \mathrm{H}_{23} \mathrm{NO}_{4} \mathrm{~S}\right]^{+}: m / z 374.1421$, found 374.1413.

2-[(2S,4R)-4-Benzyl-3-tosyloxazolidin-2-yl]-1-phenylethanone (5f). 71\% yield; white solid; IR $\left(\mathrm{CH}_{2} \mathrm{Cl}_{2}\right) v_{\max } 3060.0,3027.9,2924.2,1687.4,1597.2,1349.6,1163.3 \mathrm{~cm}^{-1} ;{ }^{1} \mathrm{H}-\mathrm{NMR}$ (400 MHz, $\left.\mathrm{CDCl}_{3}\right) \delta 7.92(\mathrm{~d}, J=7.2 \mathrm{~Hz}, 2 \mathrm{H}), 7.80(\mathrm{~d}, J=8.4 \mathrm{~Hz}, 2 \mathrm{H}), 7.24-7.58(\mathrm{~m}, 9 \mathrm{H}), 5.55(\mathrm{dd}, J=8.8$, $2.0 \mathrm{~Hz}, 1 \mathrm{H}), 3.89$ (ddd, $J=9.4,6.2,3.4 \mathrm{~Hz}, 1 \mathrm{H}), 3.75(\mathrm{dd}, J=9.6,3.2 \mathrm{~Hz}, 1 \mathrm{H}), 3.63(\mathrm{dd}, J=16.8$, $2.0 \mathrm{~Hz}, 1 \mathrm{H}), 3.13-3.28(\mathrm{~m}, 3 \mathrm{H}), 2.97(\mathrm{dd}, J=13.4,9.4 \mathrm{~Hz}, 1 \mathrm{H}), 2.40(\mathrm{~s}, 3 \mathrm{H}) ;{ }^{13} \mathrm{C}-\mathrm{NMR}(100 \mathrm{MHz}$, $\left.\mathrm{CDCl}_{3}\right) \delta 196.6,144.5,137.1$,136.6, 133.5, 130.1, 130.0, 129.1, 128.7, 128.3, 128.0, 127.0, 89.2, 69.0, 60.2, 45.6, 40.8, 21.6; HRMS (ESI+) calcd for $\left[\mathrm{C}_{25} \mathrm{H}_{25} \mathrm{NO}_{4} \mathrm{~S}\right]^{+}: \mathrm{m} / z$ 436.1577, found 436.1576 .

2-[(2R,4R)-4-Benzyl-3-tosyloxazolidin-2-yl]-1-(3,4-dichlorophenyl)ethanone (5g). 30\% yield; white solid; IR $\left(\mathrm{CH}_{2} \mathrm{Cl}_{2}\right) v_{\max } 3063.5,3028.4,2924.0,1691.4,1597.7,1350.47,1164.0 \mathrm{~cm}^{-1}$; ${ }^{1} \mathrm{H}-\mathrm{NMR}$ $\left(400 \mathrm{MHz}, \mathrm{CDCl}_{3}\right) \delta 7.94(\mathrm{~d}, J=2.0 \mathrm{~Hz}, 1 \mathrm{H}), 7.78(\mathrm{~d}, J=8.4 \mathrm{~Hz}, 2 \mathrm{H}), 7.72(\mathrm{dd}, J=8.4,2.0 \mathrm{~Hz}, 1 \mathrm{H})$, $7.54(\mathrm{~d}, J=8.4 \mathrm{~Hz}, 2 \mathrm{H}), 7.25-7.37(\mathrm{~m}, 8 \mathrm{H}), 5.46(\mathrm{dd}, J=8.6,2.2 \mathrm{~Hz}, 1 \mathrm{H}), 3.87$ (ddd, $J=9.1,6.1$, $3.1 \mathrm{~Hz}, 1 \mathrm{H}), 3.76(\mathrm{dd}, J=9.2,3.2 \mathrm{~Hz}, 1 \mathrm{H}), 3.51(\mathrm{dd}, J=16.8,2.0 \mathrm{~Hz}, 1 \mathrm{H}), 3.28(\mathrm{dd}, J=9.2,6.4 \mathrm{~Hz}$, 1H), 2.96-3.12 (m, 3H), $2.43(\mathrm{~s}, 3 \mathrm{H}) ;{ }^{13} \mathrm{C}-\mathrm{NMR}\left(100 \mathrm{MHz}, \mathrm{CDCl}_{3}\right) \delta 194.4,144.6,138.0,136.9$, 136.0, 133.4, 133.3, 130.8, 130.3, 130.1, 128.7, 127.9, 127.3, 127.0, 88.9, 69.0, 60.0, 45.5, 40.6, 21.6; HRMS (ESI+) calcd for $\left[\mathrm{C}_{25} \mathrm{H}_{23} \mathrm{Cl}_{2} \mathrm{NO}_{4} \mathrm{~S}\right]^{+}: m / z 504.0798$, found 504.0802.

2-[(2R,4R)-4-Benzyl-3-tosyloxazolidin-2-yl]-1-(3,4-dimethoxyphenyl)ethanone (5h). 98\% yield; yellow oil; IR $\left(\mathrm{CH}_{2} \mathrm{Cl}_{2}\right) v_{\max } 3062.0,3027.3,2936.2,1672.8,1595.7,1347.3,1163.2 \mathrm{~cm}^{-1} ;{ }^{1} \mathrm{H}-\mathrm{NMR}$ $\left(400 \mathrm{MHz}, \mathrm{CDCl}_{3}\right) \delta 7.73(\mathrm{~d}, J=8.0 \mathrm{~Hz}, 2 \mathrm{H}), 7.45-7.54(\mathrm{~m}, 2 \mathrm{H}), 7.24(\mathrm{t}, J=7.2 \mathrm{~Hz}, 2 \mathrm{H}), 7.17(\mathrm{~d}$, $J=14.0 \mathrm{~Hz}, 2 \mathrm{H}), 6.90-6.93(\mathrm{~m}, 1 \mathrm{H}), 6.84(\mathrm{~d}, J=8.4 \mathrm{~Hz}, 1 \mathrm{H}), 5.47(\mathrm{dd}, J=8.8,2.0 \mathrm{~Hz}, 1 \mathrm{H}), 3.85$ (s, $3 \mathrm{H}), 3.83(\mathrm{~s}, 3 \mathrm{H}), 3.68(\mathrm{dd}, J=9.0,3.4 \mathrm{~Hz}, 1 \mathrm{H}), 3.52(\mathrm{dd}, J=16.4,1.6 \mathrm{~Hz}, 1 \mathrm{H}), 3.19(\mathrm{dd}, J=9.2$, $6.4 \mathrm{~Hz}, 1 \mathrm{H}), 3.04-3.13(\mathrm{~m}, 1 \mathrm{H}), 2.88(\mathrm{dd}, J=13.4,9.4 \mathrm{~Hz}, 1 \mathrm{H}), 2.31(\mathrm{~s}, 3 \mathrm{H}) ;{ }^{13} \mathrm{C}-\mathrm{NMR}(100 \mathrm{MHz}$, $\left.\mathrm{CDCl}_{3}\right) \delta 195.1,153.6,149.0,144.4,143.0,137.5,137.3,137.0,133.4,130.1,130.0,129.9,129.8$, $126.8,110.1,89.4,68.9,56.8,56.1,55.9,45.1,40.6,21.5,21.0$; HRMS (ESI+) calcd for $\left[\mathrm{C}_{27} \mathrm{H}_{29} \mathrm{NO}_{6} \mathrm{~S}\right]^{+}: m / z$ 496.1788, found 496.1779 . 
2-[(2R,4R)-4-Benzyl-3-tosyloxazolidin-2-yl]-1-(4-fluorophenyl)ethanone (5i). 51\% yield; yellow solid; IR $\left(\mathrm{CH}_{2} \mathrm{Cl}_{2}\right) v_{\max } 3063.5,3028.6,2924.9,1686.4,1597.2,1350.0,1163.9 \mathrm{~cm}^{-1} ;{ }^{1} \mathrm{H}-\mathrm{NMR}(400 \mathrm{MHz}$, $\left.\mathrm{CDCl}_{3}\right) \delta 7.93(\mathrm{dd}, J=8.8,5.6 \mathrm{~Hz}, 2 \mathrm{H}), 7.79(\mathrm{~d}, J=8.0 \mathrm{~Hz}, 2 \mathrm{H}), 7.10-7.34(\mathrm{~m}, 9 \mathrm{H}), 5.50(\mathrm{dd}, J=8.8$, $2.0 \mathrm{~Hz}, 1 \mathrm{H}), 3.87$ (ddd, $J=9.3,6.3,3.1 \mathrm{~Hz}, 1 \mathrm{H}), 3.75$ (dd, $J=9.2,3.2 \mathrm{~Hz}, 1 \mathrm{H}), 3.52$ (dd, $J=16.8$, $2.0 \mathrm{~Hz}, 1 \mathrm{H}), 3.25(\mathrm{dd}, J=9.2,6.0 \mathrm{~Hz}, 1 \mathrm{H}), 2.98-3.18(\mathrm{~m}, 2 \mathrm{H}), 2.95(\mathrm{dd}, J=13.6,9.6 \mathrm{~Hz}, 1 \mathrm{H}), 2.40$ $(\mathrm{s}, 3 \mathrm{H}) ;{ }^{13} \mathrm{C}-\mathrm{NMR}\left(100 \mathrm{MHz}, \mathrm{CDCl}_{3}\right) \delta 195.0,167.2,164.7,144.5,137.0,133.5,133.1,131.1,131.0$, 130.2, 130.0, 128.7, 128.0, 127.0, 115.8, 89.2, 69.0, 60.2, 45.5, 40.8, 21.6; HRMS (ESI+) calcd for $\left[\mathrm{C}_{25} \mathrm{H}_{24} \mathrm{FNO}_{4} \mathrm{~S}\right]^{+}: \mathrm{m} / z$ 454.1483, found 454.1489.

2-[(2R,4R)-4-Benzyl-3-tosyloxazolidin-2-yl]-1-(naphth-1-yl)ethanone (5j). 96\% yield; yellow oil; IR $\left(\mathrm{CH}_{2} \mathrm{Cl}_{2}\right) v_{\max } 3060.6,3028.6,2924.4,1678.7,1596.3,1349.6,1163.4 \mathrm{~cm}^{-1} ;{ }^{1} \mathrm{H}-\mathrm{NMR}$ (400 MHz, $\left.\mathrm{CDCl}_{3}\right) \delta 8.82(\mathrm{~d}, J=8.4 \mathrm{~Hz}, 1 \mathrm{H}), 7.99(\mathrm{~d}, J=8.0 \mathrm{~Hz}, 1 \mathrm{H}), 7.84-7.90(\mathrm{~m}, 4 \mathrm{H}), 7.50-7.62(\mathrm{~m}, 3 \mathrm{H})$, $7.22-7.35(\mathrm{~m}, 7 \mathrm{H}), 5.66(\mathrm{dd}, J=8.6,2.2 \mathrm{~Hz}, 1 \mathrm{H}), 3.94(\mathrm{ddd}, J=9.3,6.3,3.1 \mathrm{~Hz}, 1 \mathrm{H}), 3.78-3.85$ $(\mathrm{m}, 2 \mathrm{H}), 3.34(\mathrm{dd}, J=16.8,8.8 \mathrm{~Hz}, 1 \mathrm{H}), 3.28(\mathrm{dd}, J=9.2,6.0 \mathrm{~Hz}, 1 \mathrm{H}), 3.18(\mathrm{dd}, J=13.4,3.4 \mathrm{~Hz}, 1 \mathrm{H})$, $2.98(\mathrm{dd}, J=13.6,9.2 \mathrm{~Hz}, 1 \mathrm{H}), 2.36(\mathrm{~s}, 3 \mathrm{H}) ;{ }^{13} \mathrm{C}-\mathrm{NMR}\left(100 \mathrm{MHz}, \mathrm{CDCl}_{3}\right) \delta 200.1,144.5,137.1$, $134.9,134.0,133.6,133.4,130.3,130.2,130.0,128.9,128.7,128.6,128.2,128.0,127.0,126.6,126.0$, 127.5, 89.7, 69.1, 60.4, 48.8, 40.9, 21.6; HRMS (ESI+) calcd for $\left[\mathrm{C}_{29} \mathrm{H}_{27} \mathrm{NO}_{4} \mathrm{~S}\right]^{+}: \mathrm{m} / z$ 486.1734, found 486.1729 .

2-[(2R,4R)-4-Benzyl-3-tosyloxazolidin-2-yl]-1-(thien-2-yl)ethanone (5k). 41\% yield; brown solid; IR $\left(\mathrm{CH}_{2} \mathrm{Cl}_{2}\right) v_{\max } 3105.0,3028.7,2918.5,1660.9,1597.4,1350.3,1163.1 \mathrm{~cm}^{-1} ;{ }^{1} \mathrm{H}-\mathrm{NMR}(400 \mathrm{MHz}$, $\left.\mathrm{CDCl}_{3}\right) \delta 7.79(\mathrm{~d}, J=8.0 \mathrm{~Hz}, 2 \mathrm{H}), 7.69(\mathrm{dd}, J=3.8,1.0 \mathrm{~Hz}, 1 \mathrm{H}), 7.66(\mathrm{dd}, J=5.0,1.0 \mathrm{~Hz}, 1 \mathrm{H}), 7.33$ (t, $J=7.2 \mathrm{~Hz}, 4 \mathrm{H}), 7.25(\mathrm{~d}, J=6.8 \mathrm{~Hz}, 3 \mathrm{H}), 7.14(\mathrm{t}, J=2.6 \mathrm{~Hz}, 1 \mathrm{H}), 5.47(\mathrm{dd}, J=8.6,2.2 \mathrm{~Hz}, 1 \mathrm{H})$, $3.86(\mathrm{ddd}, J=9.4,6.2,3.2 \mathrm{~Hz}, 1 \mathrm{H}), 3.76(\mathrm{dd}, J=9.2,3.2 \mathrm{~Hz}, 1 \mathrm{H}), 3.54(\mathrm{dd}, J=16.2,2.2 \mathrm{~Hz}, 1 \mathrm{H})$, $3.25(\mathrm{dd}, J=9.2,6.4 \mathrm{~Hz}, 1 \mathrm{H}), 3.09-3.15(\mathrm{~m}, 2 \mathrm{H}), 2.94(\mathrm{dd}, J=13.6,9.6 \mathrm{~Hz}, 1 \mathrm{H}), 2.42(\mathrm{~s}, 3 \mathrm{H})$; ${ }^{13} \mathrm{C}$ - NMR (100 MHz, $\left.\mathrm{CDCl}_{3}\right) \delta 189.2,144.6,144.1,137.1,134.5,134.4$,133.5, 132.9, 130.2, 130.0, 128.7, 128.3, 128.0, 127.0, 89.2, 69.0, 60.2, 46.2, 40.7, 21.6; HRMS (ESI+) calcd for $\left[\mathrm{C}_{23} \mathrm{H}_{23} \mathrm{NO}_{4} \mathrm{~S}_{2}\right]^{+}$: $\mathrm{m} / \mathrm{z} 442.1141$, found 442.1119 .

Methyl 2-[(2S,4R)-4-(hydroxymethyl)-3-tosyloxazolidin-2-yl]acetate (6a). 38\% yield; yellow oil; IR $\left(\mathrm{CH}_{2} \mathrm{Cl}_{2}\right) v_{\max }$ 3532.4, 2953.5, 1738.3. 1597.5. 1349.3. $1164.0 \mathrm{~cm}^{-1} ;{ }^{1} \mathrm{H}-\mathrm{NMR}\left(400 \mathrm{MHz}, \mathrm{CDCl}_{3}\right)$ $\delta 7.73(\mathrm{~d}, J=8.4 \mathrm{~Hz}, 2 \mathrm{H}), 7.34(\mathrm{~d}, J=8.4 \mathrm{~Hz}, 2 \mathrm{H}), 5.31(\mathrm{dd}, J=8.2,3.4 \mathrm{~Hz}, 1 \mathrm{H}), 3.87$ (dd, $J=9.0$, $3.4 \mathrm{~Hz}, 1 \mathrm{H}), 3.63-3.69$ (m, 6H), 3.44 (dd, $J=9.0,6.2 \mathrm{~Hz}, 1 \mathrm{H}), 3.03(\mathrm{dd}, J=15.6,3.6 \mathrm{~Hz}, 1 \mathrm{H}), 2.75$ $(\mathrm{dd}, J=16.0,8.0 \mathrm{~Hz}, 1 \mathrm{H}), 2.41(\mathrm{~s}, 3 \mathrm{H}) ;{ }^{13} \mathrm{C}-\mathrm{NMR}\left(100 \mathrm{MHz}, \mathrm{CDCl}_{3}\right) \delta 170.0,144.8,133.0,130.2$, 128.0, 127.0, 89.1, 67.6, 63.1, 60.2, 52.0, 41.3, 21.6; HRMS (ESI+) calcd for $\left[\mathrm{C}_{14} \mathrm{H}_{19} \mathrm{NO}_{6} \mathrm{~S}\right]^{+}: \mathrm{m} / z$ 330.1006 , found 330.0995 .

Ethyl 2-[(2S,4R)-4-(hydroxymethyl)-3-tosyloxazolidin-2-yl]acetate (6b). 50\% yield; brown oil; IR $\left(\mathrm{CH}_{2} \mathrm{Cl}_{2}\right) v_{\max } 3531.9,2982.6,1732.1,1597.6,1350.5,1164.6 \mathrm{~cm}^{-1} ;{ }^{1} \mathrm{H}-\mathrm{NMR}\left(400 \mathrm{MHz}, \mathrm{CDCl}_{3}\right)$ $\delta 7.73(\mathrm{~d}, J=8.4 \mathrm{~Hz}, 2 \mathrm{H}), 7.33(\mathrm{dd}, J=8.0 \mathrm{~Hz}, 2 \mathrm{H}), 5.31(\mathrm{dd}, J=8.2,3.4 \mathrm{~Hz}, 1 \mathrm{H}), 4.14(\mathrm{q}, J=7.2$ $\mathrm{Hz}, 2 \mathrm{H}), 3.87(\mathrm{dd}, J=9.4,3.4 \mathrm{~Hz}, 1 \mathrm{H}), 3.63-3.70(\mathrm{~m}, 3 \mathrm{H}), 3.44(\mathrm{dd}, J=9.0,6.2 \mathrm{~Hz}, 1 \mathrm{H}), 3.01$ (dd, $J=15.8,3.4 \mathrm{~Hz}, 2 \mathrm{H}), 2.73(\mathrm{dd}, J=15.8,8.2 \mathrm{~Hz}, 1 \mathrm{H}), 2.37-2.41(\mathrm{~m}, 4 \mathrm{H}), 1.24(\mathrm{t}, J=7.2 \mathrm{~Hz}, 3 \mathrm{H}) ;{ }^{13} \mathrm{C}-$ 
NMR (100 MHz, $\left.\mathrm{CDCl}_{3}\right) \delta 169.6,144.8,133.1,130.1,128.0,89.1,67.6,63.2,61.0,60.2,41.5,21.6$, 14.1; HRMS (ESI+) calcd for $\left[\mathrm{C}_{15} \mathrm{H}_{21} \mathrm{NO}_{6} \mathrm{~S}\right]^{+}: \mathrm{m} / z$ 344.1162, found 344.1186 .

Benzyl 2-[(2S,4R)-4-(hydroxymethyl)-3-tosyloxazolidin-2-yl]acetate (6c). 37\% yield; brown oil; IR $\left(\mathrm{CH}_{2} \mathrm{Cl}_{2}\right) v_{\max } 3532.5,3064.1,2949.6,1736.5,1597.4,1350.1,1164.3 \mathrm{~cm}^{-1} ;{ }^{1} \mathrm{H}-\mathrm{NMR}(400 \mathrm{MHz}$, $\left.\mathrm{CDCl}_{3}\right) \delta 7.75(\mathrm{~d}, J=8.0 \mathrm{~Hz}, 2 \mathrm{H}), 7.33-7.37(\mathrm{~m}, 7 \mathrm{H}), 5.38(\mathrm{dd}, J=8.0,3.6 \mathrm{~Hz}, 1 \mathrm{H}), 5.16(\mathrm{~s}, 2 \mathrm{H}), 3.89$ $(\mathrm{dd}, J=9.2,3.6 \mathrm{~Hz}, 1 \mathrm{H}), 3.64-3.73(\mathrm{~m}, 3 \mathrm{H}), 3.46(\mathrm{dd}, J=9.2,6.4 \mathrm{~Hz}, 1 \mathrm{H}), 3.10(\mathrm{dd}, J=15.6,3.6 \mathrm{~Hz}$, 1H), $2.83(\mathrm{dd}, J=15.6,8.0 \mathrm{~Hz}, 1 \mathrm{H}), 2.42(\mathrm{~s}, 3 \mathrm{H}) ;{ }^{13} \mathrm{C}-\mathrm{NMR}\left(100 \mathrm{MHz}, \mathrm{CDCl}_{3}\right) \delta 169.5,144.8,141.0$, 135.5, 133.1, 130.2, 128.6, 128.3, 128.0, 127.0, 89.1, 67.6, 66.8, 65.1, 63.2, 60.2, 41.5, 21.6; HRMS (ESI+) calcd for $\left[\mathrm{C}_{20} \mathrm{H}_{23} \mathrm{NO}_{6} \mathrm{~S}\right]^{+}: m / z 406.1319$, found 406.1327 .

Phenyl 2-[(2S,4R)-4-(hydroxymethyl)-3-tosyloxazolidin-2-yl]acetate (6d). 8\% yield; yellow oil; IR $\left(\mathrm{CH}_{2} \mathrm{Cl}_{2}\right) v_{\max } 3515.0,3064.8,2526.4,1755.0,1594.6,1324.6,1163.1 \mathrm{~cm}^{-1} ;{ }^{1} \mathrm{H}-\mathrm{NMR}(400 \mathrm{MHz}$, $\left.\mathrm{CDCl}_{3}\right) \delta 7.77(\mathrm{~d}, J=3.2 \mathrm{~Hz}, 2 \mathrm{H}), 7.74(\mathrm{~d}, J=4.4 \mathrm{~Hz}, 2 \mathrm{H}), 7.05-7.15(\mathrm{~m}, 5 \mathrm{H}), 5.48(\mathrm{dd}, J=7.6$, $4.0 \mathrm{~Hz}, 1 \mathrm{H}), 3.95(\mathrm{dd}, J=9.0,3.8 \mathrm{~Hz}, 1 \mathrm{H}), 3.72-3.76(\mathrm{~m}, 2 \mathrm{H}), 3.46-3.57(\mathrm{~m}, 3 \mathrm{H}), 3.31$ (dd, $J=15.4$, $3.8 \mathrm{~Hz}, 1 \mathrm{H}), 3.03(\mathrm{dd}, J=15.6,7.6 \mathrm{~Hz}, 1 \mathrm{H}), 2.45(\mathrm{~s}, 3 \mathrm{H}) ;{ }^{13} \mathrm{C}-\mathrm{NMR}\left(100 \mathrm{MHz}, \mathrm{CDCl}_{3}\right) \delta 168.1$, 150.4, 144.9, 143.7, 133.0, 130.2, 130.1, 129.8, 129.4, 128.0, 127.1, 121.6, 118.0, 100.8, 89.1, 67.7, 63.3, 62.4, 54.1, 41.6, 21.6; HRMS (ESI+) calcd for $\left[\mathrm{C}_{19} \mathrm{H}_{21} \mathrm{NO}_{6} \mathrm{~S}^{+}: \mathrm{m} / z\right.$ 392.1162, found 392.1187.

1-[(2S,4R)-4-(Hydroxymethyl)-3-tosyloxazolidin-2-yl]propan-2-one (6e). 29\% yield; yellow oil; IR $\left(\mathrm{CH}_{2} \mathrm{Cl}_{2}\right) v_{\max } 3516.2,3060.0,2953.8,1715.3,1597.3,1348.3,1163.9 \mathrm{~cm}^{-1} ;{ }^{1} \mathrm{H}$ NMR (400 MHz, $\left.\mathrm{CDCl}_{3}\right) \delta 7.72(\mathrm{~d}, J=8.0 \mathrm{~Hz}, 2 \mathrm{H}), 7.34(\mathrm{~d}, J=8.0 \mathrm{~Hz}, 2 \mathrm{H}), 5.28(\mathrm{dd}, J=8.0,2.8 \mathrm{~Hz}, 1 \mathrm{H}), 3.84(\mathrm{dd}$, $J=10.4,2.8 \mathrm{~Hz}, 1 \mathrm{H}), 3.67(\mathrm{~s}, 3 \mathrm{H}), 3.35-3.39(\mathrm{~m}, 1 \mathrm{H}), 3.15(\mathrm{dd}, J=16.8,2.8 \mathrm{~Hz}, 1 \mathrm{H}), 2.92(\mathrm{dd}$, $J=16.8,8.0 \mathrm{~Hz}, 2 \mathrm{H}), 2.42(\mathrm{~s}, 3 \mathrm{H}), 2.19(\mathrm{~s}, 3 \mathrm{H}) ;{ }^{13} \mathrm{C} \mathrm{NMR}\left(100 \mathrm{MHz}, \mathrm{CDCl}_{3}\right) \delta 205.3,144.8,132.9$, 130.2, 128.0, 88.7, 67.7, 63.5, 60.0, 49.8, 30.9, 21.6; HRMS (ESI+) calcd for $\left[\mathrm{C}_{14} \mathrm{H}_{19} \mathrm{NO}_{5} \mathrm{~S}\right]^{+}: \mathrm{m} / \mathrm{z}$ 314.1057, found 314.1050 .

2-[(2S, 4R)-4-(Hydroxymethyl)-3-tosyloxazolidin-2-yl]-1-phenylethanone (6f). 23\% yield; brown solid; IR $\left(\mathrm{CH}_{2} \mathrm{Cl}_{2}\right) v_{\max } 3515.4,3061.5,2933.4,1687.2,1597.1,1347.7,1163.3 \mathrm{~cm}^{-1} ;{ }^{1} \mathrm{H}-\mathrm{NMR}(400 \mathrm{MHz}$, $\left.\mathrm{CDCl}_{3}\right) \delta 7.96(\mathrm{~d}, J=8.4 \mathrm{~Hz}, 2 \mathrm{H}), 7.75(\mathrm{~d}, J=8.4 \mathrm{~Hz}, 2 \mathrm{H}), 7.54-7.58(\mathrm{~m}, 1 \mathrm{H}), 7.45(\mathrm{t}, J=7.6 \mathrm{~Hz}$, $2 \mathrm{H}), 7.34(\mathrm{~d}, J=8.0 \mathrm{~Hz}, 2 \mathrm{H}), 5.53(\mathrm{dd}, J=8.4,2.4 \mathrm{~Hz}, 1 \mathrm{H}), 3.88(\mathrm{dd}, J=8.6,2.2 \mathrm{~Hz}, 1 \mathrm{H}), 3.68-3.72$ (m, 4H), $3.47(\mathrm{dd}, J=16.8,8.4 \mathrm{~Hz}, 1 \mathrm{H}), 3.39-3.42(\mathrm{~m}, 1 \mathrm{H}), 2.41(\mathrm{~s}, 3 \mathrm{H}) ;{ }^{13} \mathrm{C}-\mathrm{NMR}(100 \mathrm{MHz}$, $\left.\mathrm{CDCl}_{3}\right) \delta 196.7,144.8,136.5,133.6,133.0,130.2,130.0,128.7,128.3,128.0,89.3,67.8,63.5,60.1$, 45.5, 21.6; HRMS (ESI+) calcd for $\left[\mathrm{C}_{19} \mathrm{H}_{21} \mathrm{NO}_{5} \mathrm{~S}\right]^{+}: \mathrm{m} / z$ 376.1213, found 376.1187.

1-(3,4-Dichlorophenyl)-2-[(2S,4R)-4-(hydroxymethyl)-3-tosyloxazolidin-2-yl]ethanone $\quad(\mathbf{6 g}) . \quad 12 \%$ yield; brown oil; IR $\left(\mathrm{CH}_{2} \mathrm{Cl}_{2}\right) v_{\max }$ 3513.6, 3064.1, 2925.2, 1691.4, 1584.1, 1333.8, $1162.5 \mathrm{~cm}^{-1}$; ${ }^{1} \mathrm{H}-\mathrm{NMR}\left(400 \mathrm{MHz}, \mathrm{CDCl}_{3}\right) \delta 8.05(\mathrm{~d}, J=2.0 \mathrm{~Hz}, 1 \mathrm{H}), 7.80(\mathrm{~d}, J=7.6 \mathrm{~Hz}, 2 \mathrm{H}), 7.37(\mathrm{~d}, J=8.0 \mathrm{~Hz}$, $2 \mathrm{H}), 7.28(\mathrm{~d}, J=7.6 \mathrm{~Hz}, 2 \mathrm{H}), 5.48(\mathrm{dd}, J=8.2,2.6 \mathrm{~Hz}, 1 \mathrm{H}), 3.88(\mathrm{dd}, J=8.6,2.2 \mathrm{~Hz}, 1 \mathrm{H}), 3.66-3.77$ $(\mathrm{m}, 7 \mathrm{H}), 3.39-3.45(\mathrm{~m}, 2 \mathrm{H}), 2.45(\mathrm{~s}, 3 \mathrm{H}), 2.40-2.42(\mathrm{~m}, 4 \mathrm{H}) ;{ }^{13} \mathrm{C}-\mathrm{NMR}\left(100 \mathrm{MHz}, \mathrm{CDCl}_{3}\right) \delta 194.4$, 145.0, 144.2, 138.2, 136.1, 133.5, 132.8, 131.0, 130.9, 128.0, 127.3, 127.2, 126.9, 126.4, 89.1, 60.1, 53.4, 21.6; HRMS (ESI+) calcd for $\left[\mathrm{C}_{19} \mathrm{H}_{19} \mathrm{Cl}_{2} \mathrm{NO}_{5} \mathrm{~S}\right]^{+}: \mathrm{m} / z$ 444.0434, found 444.0388. 
1-(3,4-Dimethoxyphenyl)-2-[(2S,4R)-4-(hydroxymethyl)-3-tosyloxazolidin-2-yl]ethanone (6h). 38\% yield; yellow oil; IR $\left(\mathrm{CH}_{2} \mathrm{Cl}_{2}\right) v_{\max } 3268.9,3060.0,2966.1,1673.8,1595.8,1334.0,1149.5 \mathrm{~cm}^{-1}$; ${ }^{1} \mathrm{H}-\mathrm{NMR}$ $\left(400 \mathrm{MHz}, \mathrm{CDCl}_{3}\right) \delta 7.73(\mathrm{~d}, J=8.4 \mathrm{~Hz}, 2 \mathrm{H}), 7.49(\mathrm{dd}, J=8.4,2.0 \mathrm{~Hz}, 1 \mathrm{H}), 7.41(\mathrm{~d}, J=2.0 \mathrm{~Hz}, 1 \mathrm{H})$, $7.22(\mathrm{~d}, J=8.0 \mathrm{~Hz}, 2 \mathrm{H}), 6.81(\mathrm{~d}, J=8.4 \mathrm{~Hz}, 1 \mathrm{H}), 6.07(\mathrm{~d}, J=9.6 \mathrm{~Hz}, 1 \mathrm{H}), 5.13(\mathrm{t}, J=5.0 \mathrm{~Hz}, 1 \mathrm{H})$, $3.88(\mathrm{~s}, 3 \mathrm{H}), 3.73-3.84(\mathrm{~m}, 6 \mathrm{H}), 3.27(\mathrm{~d}, J=9.6 \mathrm{~Hz}, 1 \mathrm{H}), 3.22(\mathrm{~d}, J=4.8 \mathrm{~Hz}, 2 \mathrm{H}), 2.34(\mathrm{~s}, 3 \mathrm{H})$; ${ }^{13} \mathrm{C}-\mathrm{NMR}\left(100 \mathrm{MHz}, \mathrm{CDCl}_{3}\right) \delta$ 194.6, 194.5, 153.6, 149.0, 148.9, 143.7, 143.5, 138.1, 137.7, 130.0, 129.8, 127.0, 126.8, 123.4, 110.1, 100.0, 70.5, 56.0, 47.8, 45.3, 43.1, 42.8, 21.5; HRMS (ESI+) calcd for $\left[\mathrm{C}_{21} \mathrm{H}_{25} \mathrm{NO}_{7} \mathrm{~S}\right]^{+}: \mathrm{m} / z$ 436.1425, found 436.1418 .

1-(4-Fluorophenyl)-2-[(2S,4R)-4-(hydroxymethyl)-3-tosyloxazolidin-2-yl]ethanone (6i). 23\% yield; brown oil; IR $\left(\mathrm{CH}_{2} \mathrm{Cl}_{2}\right) v_{\max } 3515.7,3064.1,2925.2,1687.0,1597.5,1346.7,1163.5 \mathrm{~cm}^{-1} ;{ }^{1} \mathrm{H}-\mathrm{NMR}$ $\left(400 \mathrm{MHz}, \mathrm{CDCl}_{3}\right) \delta 8.00(\mathrm{dd}, J=8.8,5.6 \mathrm{~Hz}, 2 \mathrm{H}), 7.75(\mathrm{~d}, J=8.4 \mathrm{~Hz}, 2 \mathrm{H}), 7.35(\mathrm{~d}, J=8.0 \mathrm{~Hz}, 2 \mathrm{H})$, $7.12(\mathrm{t}, J=8.6 \mathrm{~Hz}, 2 \mathrm{H}), 5.51(\mathrm{dd}, J=8.4,2.4 \mathrm{~Hz}, 1 \mathrm{H}), 3.88(\mathrm{dd}, J=8.4,2.0 \mathrm{~Hz}, 1 \mathrm{H}), 3.65-3.74$ $(\mathrm{m}, 5 \mathrm{H}), 3.39-3.48(\mathrm{~m}, 2 \mathrm{H}), 2.43(\mathrm{~s}, 3 \mathrm{H}), 2.38-2.41(\mathrm{~m}, 1 \mathrm{H}) ;{ }^{13} \mathrm{C}-\mathrm{NMR}\left(100 \mathrm{MHz}, \mathrm{CDCl}_{3}\right) \delta 195.1$, 144.9, 132.9, 132.4, 132.3, 131.1, 131.0, 130.2, 129.1, 128.0, 89.3, 67.8, 63.4, 60.1, 45.4, 22.6, 21.6; HRMS (ESI+) calcd for $\left[\mathrm{C}_{19} \mathrm{H}_{20} \mathrm{FNO}_{5} \mathrm{~S}\right]^{+}: \mathrm{m} / z$ 394.1119, found 394.1100.

2-[(2S,4R)-4-(Hydroxymethyl)-3-tosyloxazolidin-2-yl]-1-(naphth-1-yl)ethanone (6j). 24\% yield; brown oil; IR $\left(\mathrm{CH}_{2} \mathrm{Cl}_{2}\right) v_{\max } 3515.8,3052.0,2957.9,1678.1,1509.0,1346.9,1163.1 \mathrm{~cm}^{-1}$; ${ }^{1} \mathrm{H}-\mathrm{NMR}$ $\left(400 \mathrm{MHz}, \mathrm{CDCl}_{3}\right) \delta 8.70(\mathrm{~d}, J=8.0 \mathrm{~Hz}, 1 \mathrm{H}), 7.98(\mathrm{dd}, J=9.0,4.6 \mathrm{~Hz}, 2 \mathrm{H}), 7.77(\mathrm{~d}, J=8.4 \mathrm{~Hz}, 2 \mathrm{H})$, $7.48-7.60(\mathrm{~m}, 4 \mathrm{H}), 7.33(\mathrm{~d}, J=8.0 \mathrm{~Hz}, 2 \mathrm{H}), 5.58(\mathrm{dd}, J=8.4,2.8 \mathrm{~Hz}, 1 \mathrm{H}), 3.86-3.91(\mathrm{~m}, 2 \mathrm{H})$, $3.65-3.78(\mathrm{~m}, 4 \mathrm{H}), 3.52(\mathrm{dd}, J=16.4,8.4 \mathrm{~Hz}, 1 \mathrm{H}), 3.42(\mathrm{dd}, J=9.0,6.2 \mathrm{~Hz}, 1 \mathrm{H}), 2.41(\mathrm{~s}, 3 \mathrm{H})$; ${ }^{13} \mathrm{C}-\mathrm{NMR}\left(100 \mathrm{MHz}, \mathrm{CDCl}_{3}\right) \delta$ 200.2, 144.8, 134.9, 134.0, 133.8, 133.4, 133.0, 130.2, 128.7, 128.6, 128.5, 125.9, 89.8, 76.8, 60.4, 48.7, 21.5, 21.1; HRMS (ESI+) calcd for $\left[\mathrm{C}_{23} \mathrm{H}_{23} \mathrm{NO}_{5} \mathrm{~S}\right]^{+}: \mathrm{m} / z$ 426.1370, found 426.1365 .

2-[(2R,4R)-4-(Hydroxymethyl)-3-tosyloxazolidin-2-yl]-1-(thien-2-yl)ethanone (6k). 58\% yield; brown oil; IR $\left(\mathrm{CH}_{2} \mathrm{Cl}_{2}\right) v_{\max } 3270.0,3096.8,2974.2,1658.6,1597.8,1332.4,1162.3 \mathrm{~cm}^{-1}$; ${ }^{1} \mathrm{H}-\mathrm{NMR}$ $\left(400 \mathrm{MHz}, \mathrm{CDCl}_{3}\right) \delta 7.75(\mathrm{dd}, J=8.2,2.2 \mathrm{~Hz}, 2 \mathrm{H}), 7.67(\mathrm{dd}, J=3.6,1.2 \mathrm{~Hz}, 1 \mathrm{H}), 7.61(\mathrm{~d}, J=4.8 \mathrm{~Hz}$, $1 \mathrm{H}), 7.25(\mathrm{~d}, J=9.6 \mathrm{~Hz}, 2 \mathrm{H}), 7.05-7.07(\mathrm{~m}, 1 \mathrm{H}), 6.08(\mathrm{~d}, J=8.0 \mathrm{~Hz}, 1 \mathrm{H}), 5.11(\mathrm{t}, J=4.4 \mathrm{~Hz}, 1 \mathrm{H})$, $3.78(\mathrm{q}, J=13.9 \mathrm{~Hz}, 3 \mathrm{H}), 3.20(\mathrm{~d}, J=5.2 \mathrm{~Hz}, 2 \mathrm{H}), 2.36(\mathrm{~s}, 3 \mathrm{H}) ;{ }^{13} \mathrm{C}-\mathrm{NMR}\left(100 \mathrm{MHz}, \mathrm{CDCl}_{3}\right) \delta 188.7$, 144.2, 143.8, 143.6, 137.6, 134.6, 133.2, 133.1, 129.9, 128.4, 127.0, 99.4, 70.5, 69.9, 47.7, 44.3, 21.5; HRMS (ESI+) calcd for $\left[\mathrm{C}_{17} \mathrm{H}_{19} \mathrm{NO}_{5} \mathrm{~S}_{2}\right]^{+}: \mathrm{m} / z$ 382.0777, found 382.0781 .

Methyl 2-\{(2S,3aS,7aS)-3-tosyloctahydrobenzo[d]oxazol-2-yl\}acetate (7a). 15\% yield; white oil; IR $\left(\mathrm{CH}_{2} \mathrm{Cl}_{2}\right) v_{\max } 2916.8,2848.7,1715.4,1631.7,1349.0,1160.9 \mathrm{~cm}^{-1} ;{ }^{1} \mathrm{H}-\mathrm{NMR}\left(400 \mathrm{MHz}, \mathrm{CDCl}_{3}\right)$ $\delta 8.38(\mathrm{~d}, J=8.0 \mathrm{~Hz}, 2 \mathrm{H}), 8.13(\mathrm{~d}, J=8.0 \mathrm{~Hz}, 2 \mathrm{H}), 5.26(\mathrm{dd}, J=8.0,4.0 \mathrm{~Hz}, 1 \mathrm{H}), 3.68(\mathrm{~s}, 3 \mathrm{H})$, $3.42-3.48(\mathrm{~m}, 1 \mathrm{H}), 3.15(\mathrm{ddd}, J=13.0,9.0,3.0 \mathrm{~Hz}, 1 \mathrm{H}), 2.74(\mathrm{dd}, J=16.0,4.0 \mathrm{~Hz}, 1 \mathrm{H}), 2.46(\mathrm{~s}, 3 \mathrm{H})$, 2.29-2.34 (m, 1H), 1.96-1.98 (m, 1H), 1.77-1.81 (m, 2H), 1.40-1.43 (m, 1H), 1.30-1.35 (m, 4H); ${ }^{13} \mathrm{C}-\mathrm{NMR}\left(100 \mathrm{MHz}, \mathrm{CDCl}_{3}\right) \delta 171.4,152.2,147.5,143.2,136.5,127.2,121.1,118.0,110.0,88.1$, 60.2, 50.9, 39.3, 37.4, 29.7, 21.4; GCMS (EI+) calcd for $\left[\mathrm{C}_{17} \mathrm{H}_{23} \mathrm{NO}_{5} \mathrm{~S}\right]: \mathrm{m} / z$ 354.4, found 355.0. 
Ethyl 2-\{(2S,3aS,7aS)-3-tosyloctahydrobenzo[d]oxazol-2-yl\}acetate (7b). 37\% yield; white oil; IR $\left(\mathrm{CH}_{2} \mathrm{Cl}_{2}\right) v_{\max } 2942.9,1736.4,1598.4,1352.7,1162.7 \mathrm{~cm}^{-1} ;{ }^{1} \mathrm{H}-\mathrm{NMR}\left(400 \mathrm{MHz}, \mathrm{CDCl}_{3}\right) \delta 7.68(\mathrm{~d}$, $J=8.0 \mathrm{~Hz}, 2 \mathrm{H}), 7.34(\mathrm{~d}, J=8.0 \mathrm{~Hz}, 2 \mathrm{H}), 5.32(\mathrm{dd}, J=9.6,2.4 \mathrm{~Hz}, 1 \mathrm{H}), 4.15(\mathrm{q}, J=7.2 \mathrm{~Hz}, 2 \mathrm{H})$; ${ }^{13} \mathrm{C}-\mathrm{NMR}\left(100 \mathrm{MHz}, \mathrm{CDCl}_{3}\right) \delta 169.8,144.4,131.7,130.0,129.8,128.2,127.4,88.3,80.2,64.5,63.3$, $60.7,41.8,29.6,29.0,24.4,23.8,23.3,23.1,21.6,14.2$; HRMS (ESI+) calcd for $\left[\mathrm{C}_{18} \mathrm{H}_{25} \mathrm{NO}_{5} \mathrm{~S}\right]^{+}: \mathrm{m} / \mathrm{z}$ 368.1526 , found 368.1529 .

Benzyl 2-\{(2S,3aS,7aS)-3-tosyloctahydrobenzo[d]oxazol-2-yl\}acetate (7c). 19\% yield; yellow oil; IR $\left(\mathrm{CH}_{2} \mathrm{Cl}_{2}\right) v_{\max } 3032.7,2940.8,2862.9,1710.7,1620.9,1320.5,1121.0 \mathrm{~cm}^{-1} ;{ }^{1} \mathrm{H}-\mathrm{NMR}(400 \mathrm{MHz}$, $\left.\mathrm{CDCl}_{3}\right) \delta 7.74(\mathrm{~d}, J=8.0 \mathrm{~Hz}, 2 \mathrm{H}), 7.36-7.38(\mathrm{~m}, 5 \mathrm{H}), 7.23(\mathrm{~d}, J=8.0 \mathrm{~Hz}, 2 \mathrm{H}), 5.10(\mathrm{~s}, 1 \mathrm{H}), 5.07$ $(\mathrm{s}, 1 \mathrm{H}), 4.88(\mathrm{~s}, 1 \mathrm{H}), 3.67(\mathrm{ddd}, J=10.0,6.0,2.0 \mathrm{~Hz}, 1 \mathrm{H}), 3.42(\mathrm{t}, J=8.0 \mathrm{~Hz}, 1 \mathrm{H}), 3.06-3.11(\mathrm{~m}, 1 \mathrm{H})$, $2.78(\mathrm{t}, J=8.0 \mathrm{~Hz}, 1 \mathrm{H}), 2.39(\mathrm{~s}, 3 \mathrm{H}), 2.25-2.27(\mathrm{~m}, 1 \mathrm{H}), 1.98-2.02(\mathrm{~m}, 1 \mathrm{H}), 1.74-1.83(\mathrm{~m}, 1 \mathrm{H})$, $1.66-1.70(\mathrm{~m}, 1 \mathrm{H}), 1.53-1.61(\mathrm{~m}, 1 \mathrm{H}), 1.24-1.31(\mathrm{~m}, 3 \mathrm{H}) ;{ }^{13} \mathrm{C}-\mathrm{NMR}\left(100 \mathrm{MHz}, \mathrm{CDCl}_{3}\right) \delta 169.9$, 167.1, 167.0, 163.4, 160.6, 149.1, 145.1, 144.9, 143.1, 137.1, 136.4, 136.1, 135.5, 135.3, 135.2, 130.0, 129.9, 129.5, 128.4, 127.3, 102.9, 102.8, 98.0, 79.6, 77.3, 67.3, 67.1, 66.2, 65.6, 55.8, 51.5, 32.2, 31.2, 29.6, 28.8, 28.0, 25.0, 23.2, 21.7, 21.5; GCMS (EI+) calcd for $\left[\mathrm{C}_{23} \mathrm{H}_{27} \mathrm{NO}_{5} \mathrm{~S}\right]: \mathrm{m} / z$ 429.5, found 430.0.

Phenyl 2-\{(2S,3aS, 7aS)-3-tosyloctahydrobenzo[d]oxazol-2-yl\}acetate (7d). 13\% yield; white solid; IR $\left(\mathrm{CH}_{2} \mathrm{Cl}_{2}\right) v_{\max }$ 3056.0, 2943.7, 1758.4, 1597.1, 1352.8, $1163.5 \mathrm{~cm}^{-1} ;{ }^{1} \mathrm{H}-\mathrm{NMR}\left(400 \mathrm{MHz}, \mathrm{CDCl}_{3}\right)$ $\delta 7.73(\mathrm{~d}, J=8.4 \mathrm{~Hz}, 2 \mathrm{H}), 7.36(\mathrm{~d}, J=8.4 \mathrm{~Hz}, 4 \mathrm{H}), 7.21-7.25(\mathrm{~m}, 1 \mathrm{H}), 7.12(\mathrm{dd}, J=5.0,3.8 \mathrm{~Hz}, 2 \mathrm{H})$, $5.44(\mathrm{dd}, J=9.4,2.6 \mathrm{~Hz}, 1 \mathrm{H}), 3.58-3.61(\mathrm{~m}, 1 \mathrm{H}), 3.38(\mathrm{dd}, J=16.2,2.6 \mathrm{~Hz}, 1 \mathrm{H}), 3.05(\mathrm{dd}, J=16.2$, $9.0 \mathrm{~Hz}, 1 \mathrm{H}), 2.45-2.50(\mathrm{~m}, 4 \mathrm{H}), 2.37(\mathrm{ddd}, J=12.0,8.0,4.0 \mathrm{~Hz}, 1 \mathrm{H}), 1.84-2.07(\mathrm{~m}, 1 \mathrm{H}), 1.81-1.84$ $(\mathrm{m}, 2 \mathrm{H}), 1.51(\mathrm{dd}, J=12.0,3.6 \mathrm{~Hz}, 1 \mathrm{H}), 1.24-1.32(\mathrm{~m}, 4 \mathrm{H}), 0.86-0.90(\mathrm{~m}, 1 \mathrm{H}) ;{ }^{13} \mathrm{C}-\mathrm{NMR}(100 \mathrm{MHz}$, $\left.\mathrm{CDCl}_{3}\right) \delta 168.3,150.5,144.5,131.6,130.0,129.4,129.1,128.2,126.0,121.6,88.2,80.6,64.6,42.1$, 29.7, 29.1, 23.8, 23.3, 21.6; HRMS (ESI+) calcd for $\left[\mathrm{C}_{22} \mathrm{H}_{25} \mathrm{NO}_{5} \mathrm{~S}\right]^{+}: \mathrm{m} / z$ 416.1526, found 416.1525.

1-\{(2S,3aS, 7aS)-3-Tosyloctahydrobenzo[d] oxazol-2-yl\}propan-2-one (7e). 33\% yield; yellow solid; IR $\left(\mathrm{CH}_{2} \mathrm{Cl}_{2}\right) v_{\max } 3060.9,2943.0,1717.2,1598.3,1351.3,1162.1 \mathrm{~cm}^{-1} ;{ }^{1} \mathrm{H}-\mathrm{NMR}\left(400 \mathrm{MHz}, \mathrm{CDCl}_{3}\right)$ $\delta 7.63(\mathrm{~d}, J=8.4 \mathrm{~Hz}, 2 \mathrm{H}), 7.32(\mathrm{~d}, J=8.0 \mathrm{~Hz}, 2 \mathrm{H}), 5.30(\mathrm{dd}, J=9.2,2.0 \mathrm{~Hz}, 1 \mathrm{H}), 3.42$ (ddd, $J=10.9$, 9.1, $2.7 \mathrm{~Hz}, 1 \mathrm{H}), 3.13(\mathrm{dd}, J=13.6,6.0 \mathrm{~Hz}, 1 \mathrm{H}), 2.95(\mathrm{dd}, J=17.2,9.2 \mathrm{~Hz}, 1 \mathrm{H}), 2.36-2.43(\mathrm{~m}, 5 \mathrm{H})$, 2.21-2.27 (m, 1H), $2.17(\mathrm{~s}, 3 \mathrm{H}), 1.94-1.96(\mathrm{~m}, 1 \mathrm{H}), 1.71-1.77(\mathrm{~m}, 2 \mathrm{H}), 1.36-1.46(\mathrm{~m}, 1 \mathrm{H}), 1.15-1.23$ $(\mathrm{m}, 4 \mathrm{H}) ;{ }^{13} \mathrm{C}-\mathrm{NMR}\left(100 \mathrm{MHz}, \mathrm{CDCl}_{3}\right) \delta 205.4,144.4,131.5,130.0,128.1,127.3,87.7,80.3,64.3$, 50.3, 30.7, 29.6, 29.3, 29.0, 23.7, 23.3, 21.6; HRMS (ESI+) calcd for $\left[\mathrm{C}_{17} \mathrm{H}_{23} \mathrm{NO}_{4} \mathrm{~S}\right]^{+}: \mathrm{m} / z 338.1421$, found 338.1416 .

1-Phenyl-2-\{(2S,3aS, 7aS)-3-tosyloctahydrobenzo[d] oxazol-2-yl\}ethanone (7f). 32\% yield; yellow oil; IR $\left(\mathrm{CH}_{2} \mathrm{Cl}_{2}\right) v_{\max }$ 3056.0, 2942.4, 1687.0, 1597.5, 1350.5, $1162.7 \mathrm{~cm}^{-1} ;{ }^{1} \mathrm{H}-\mathrm{NMR}\left(400 \mathrm{MHz}, \mathrm{CDCl}_{3}\right)$ $\delta 7.99(\mathrm{dd}, J=8.4,1.2 \mathrm{~Hz}, 2 \mathrm{H}), 7.69(\mathrm{~d}, J=8.4 \mathrm{~Hz}, 2 \mathrm{H}), 7.54-7.58(\mathrm{~m}, 1 \mathrm{H}), 7.47$ (dd, $J=8.0,7.2 \mathrm{~Hz}$, $2 \mathrm{H}), 7.35(\mathrm{~d}, J=8.0 \mathrm{~Hz}, 2 \mathrm{H}), 5.57(\mathrm{dd}, J=9.2,2.0 \mathrm{~Hz}, 1 \mathrm{H}), 3.71(\mathrm{dd}, J=17.2,2.0 \mathrm{~Hz}, 1 \mathrm{H}), 3.48-3.54$ (m, 2H), 2.49 (dd, $J=12.6,2.2 \mathrm{~Hz}, 1 \mathrm{H}), 2.43(\mathrm{~s}, 3 \mathrm{H}), 2.32$ (ddd, $J=11.9,8.7,2.9 \mathrm{~Hz}, 1 \mathrm{H}), 1.81-2.02$ (m, 1H), $1.75-1.81(\mathrm{~m}, 2 \mathrm{H}), 1.50(\mathrm{dd}, J=12.6,3.0 \mathrm{~Hz}, 1 \mathrm{H}), 1.20-1.27$ (m, 3H); ${ }^{13} \mathrm{C}-\mathrm{NMR}(100 \mathrm{MHz}$, 
$\left.\mathrm{CDCl}_{3}\right) \delta 196.8,144.5,136.6,133.4,131.5,130.0,128.7,128.3,127.4,88.3,80.4,64.4,45.8,29.6$, 29.0, 23.8, 23.3, 21.6; HRMS (ESI+) calcd for $\left[\mathrm{C}_{22} \mathrm{H}_{25} \mathrm{NO}_{4} \mathrm{~S}\right]^{+}: \mathrm{m} / z$ 400.1577, found 400.1582 .

1-(3,4-Dichlorophenyl)-2-(3-tosyloctahydrobenzo[d]oxazol-2-yl)ethanone (7g). 18\% yield; yellow solid; IR $\left(\mathrm{CH}_{2} \mathrm{Cl}_{2}\right) v_{\max } 3068.0,2946.6,2863.7,1685.3,1583.9,1351.1,1163.7 \mathrm{~cm}^{-1}$; ${ }^{1} \mathrm{H}-\mathrm{NMR}$ $\left(400 \mathrm{MHz}, \mathrm{CDCl}_{3}\right) \delta 8.06(\mathrm{~s}, 1 \mathrm{H}), 7.81(\mathrm{dd}, J=10.0,2.0 \mathrm{~Hz}, 1 \mathrm{H}), 7.68(\mathrm{~d}, J=8.0 \mathrm{~Hz}, 2 \mathrm{H}), 7.55(\mathrm{~d}$, $J=8.0 \mathrm{~Hz}, 1 \mathrm{H}), 7.36(\mathrm{~d}, J=8.0 \mathrm{~Hz}, 2 \mathrm{H}), 5.50(\mathrm{dd}, J=10.0,2.0 \mathrm{~Hz}, 1 \mathrm{H}), 3.68(\mathrm{dd}, J=16.0,4.0 \mathrm{~Hz}$, $1 \mathrm{H}), 3.51(\mathrm{td}, J=10.0,4.0 \mathrm{~Hz}, 1 \mathrm{H}), 3.45(\mathrm{dd}, J=18.0,10.0 \mathrm{~Hz}, 1 \mathrm{H}), 2.48(\mathrm{dd}, J=14.0,2.0 \mathrm{~Hz}, 1 \mathrm{H})$, 2.44 (s, 3H), 2.31 (ddd, $J=12.0,8.0,4.0 \mathrm{~Hz}, 1 \mathrm{H}), 1.97-1.99(\mathrm{~m}, 1 \mathrm{H}), 1.76-1.82(\mathrm{~m}, 2 \mathrm{H}), 1.45-1.54$ $(\mathrm{m}, 1 \mathrm{H}), 1.20-1.27(\mathrm{~m}, 3 \mathrm{H}) ;{ }^{13} \mathrm{C}-\mathrm{NMR}\left(100 \mathrm{MHz}, \mathrm{CDCl}_{3}\right) \delta 194.6,144.6,138.0,136.1,133.4,130.9$, 130.3, 129.9, 128.3, 128.2, 127.5, 127.4, 127.3, 88.0, 80.5, 77.3, 64.3, 45.9, 29.6, 29.0, 23.8, 23.3, 21.6; GCMS (EI+) calcd for $\left[\mathrm{C}_{22} \mathrm{H}_{23} \mathrm{Cl}_{2} \mathrm{NO}_{4} \mathrm{~S}\right]: \mathrm{m} / z$ 468.4, found 468.1 .

1-(3,4-Dimethoxyphenyl)-2-\{(2S,3aS,7aS)-3-tosyloctahydrobenzo[d] oxazol-2-yl\}ethanone (7h) 93\% yield; yellow solid; IR $\left(\mathrm{CH}_{2} \mathrm{Cl}_{2}\right) \quad v_{\max } 2938.9,1672.7,1596.0,1348.4,1161.1 \mathrm{~cm}^{-1}$; ${ }^{1} \mathrm{H}-\mathrm{NMR}$ $\left(400 \mathrm{MHz}, \mathrm{CDCl}_{3}\right) \delta 7.61(\mathrm{~d}, J=8.4 \mathrm{~Hz}, 2 \mathrm{H}), 7.56(\mathrm{dd}, J=8.4,1.6 \mathrm{~Hz}, 1 \mathrm{H}), 7.46(\mathrm{~d}, J=2.0 \mathrm{~Hz}, 1 \mathrm{H})$, $7.26(\mathrm{~d}, J=8.0 \mathrm{~Hz}, 2 \mathrm{H}), 7.16(\mathrm{~d}, J=8.0 \mathrm{~Hz}, 1 \mathrm{H}), 6.83(\mathrm{~d}, J=8.4 \mathrm{~Hz}, 1 \mathrm{H}), 5.48(\mathrm{~d}, J=7.6 \mathrm{~Hz}, 1 \mathrm{H})$, $3.83(\mathrm{~s}, 3 \mathrm{H}), 3.82(\mathrm{~s}, 3 \mathrm{H}), 3.38-3.60(\mathrm{~m}, 3 \mathrm{H}), 3.21(\mathrm{ddd}, J=11.8,7.8,2.2 \mathrm{~Hz}, 1 \mathrm{H}), 2.21-2.40(\mathrm{~m}, 5 \mathrm{H})$, 1.84-1.92 (m, 2H), 1.61-1.70 (m, 3H), 1.37-1.46 (m, 2H), 1.11-1.18 (m, 4H); ${ }^{13} \mathrm{C}-\mathrm{NMR}(100 \mathrm{MHz}$, $\left.\mathrm{CDCl}_{3}\right) \delta 195.4,153.5,149.0,144.4,143.2,137.7,131.4,130.0,129.7,128.1,127.0,110.2,88.5,80.2$, 72.9, 64.3, 59.6, 56.0, 54.3, 33.3, 31.5, 29.6, 29.0, 24.6, 23.7, 21.4; HRMS (ESI+) calcd for $\left[\mathrm{C}_{22} \mathrm{H}_{23} \mathrm{Cl}_{2} \mathrm{NO}_{4} \mathrm{~S}\right]^{+}: m / z$ 468.0798, found 468.0830 .

1-(4-Fluorophenyl)-2-\{(2S,3aS,7aS)-3-tosyloctahydrobenzo[d]oxazol-2-yl\}ethanone (7i). 27\% yield; yellow solid; IR $\left(\mathrm{CH}_{2} \mathrm{Cl}_{2}\right) v_{\max } 2942.4,1687.2,1597.4,1350.7,1162.6 \mathrm{~cm}^{-1}$; ${ }^{1} \mathrm{H}-\mathrm{NMR}$ (400 MHz, $\left.\mathrm{CDCl}_{3}\right) \delta 8.01(\mathrm{t}, J=4.4 \mathrm{~Hz}, 2 \mathrm{H}), 7.69(\mathrm{~d}, J=8.0 \mathrm{~Hz}, 2 \mathrm{H}), 7.35(\mathrm{~d}, J=8.0 \mathrm{~Hz}, 2 \mathrm{H}), 7.14(\mathrm{t}$, $J=8.8 \mathrm{~Hz}, 2 \mathrm{H}), 5.53(\mathrm{dd}, J=9.2,1.6 \mathrm{~Hz}, 1 \mathrm{H}), 3.69(\mathrm{dd}, J=17.0,1.8 \mathrm{~Hz}, 1 \mathrm{H}), 3.44-3.54(\mathrm{~m}, 2 \mathrm{H})$, 2.42-2.50 (m, 5H), 2.31 (ddd, $J=11.7,8.9,3.1 \mathrm{~Hz}, 1 \mathrm{H}), 1.97-2.00(\mathrm{~m}, 1 \mathrm{H}), 1.75-1.81(\mathrm{~m}, 2 \mathrm{H}), 1.50$ (ddd, $J=18.1,12.1,6.1 \mathrm{~Hz}, 1 \mathrm{H}), 1.19-1.27$ (m, 3H); ${ }^{13} \mathrm{C}-\mathrm{NMR}\left(100 \mathrm{MHz}, \mathrm{CDCl}_{3}\right) \delta 195.2,167.1$, 164.6, 144.5, 134.3, 133.1, 133.0, 132.0, 131.7, 131.0, 128.2, 115.9, 88.2, 80.4, 64.4, 45.7, 31.5, 29.7, 23.9, 23.3, 21.6; HRMS (ESI+) calcd for $\left[\mathrm{C}_{22} \mathrm{H}_{24} \mathrm{FNO}_{4} \mathrm{~S}\right]^{+}: \mathrm{m} / z$ 418.1483, found 418.1496.

1-(Naphth-1-yl)-2-\{(2S,3aS,7aS)-3-tosyloctahydrobenzo[d]oxazol-2-yl\}ethanone (7j). 33\% yield; brown oil; IR $\left(\mathrm{CH}_{2} \mathrm{Cl}_{2}\right) v_{\max } 3056.0,2942.5,1678.0,1596.5,1350.8,1162.5 \mathrm{~cm}^{-1}$; ${ }^{1} \mathrm{H}-\mathrm{NMR}(400 \mathrm{MHz}$, $\left.\mathrm{CDCl}_{3}\right) \delta 8.75(\mathrm{~d}, J=8.4,1 \mathrm{H}), 7.99(\mathrm{~d}, J=6.8 \mathrm{~Hz}, 2 \mathrm{H}), 7.86(\mathrm{~d}, J=8.0 \mathrm{~Hz}, 1 \mathrm{H}), 7.72(\mathrm{~d}, J=8.4 \mathrm{~Hz}$, 2H), 7.51-7.61 (m, 4H), $7.33(\mathrm{~d}, J=8.0 \mathrm{~Hz}, 2 \mathrm{H}), 5.63(\mathrm{dd}, J=9.2,2.0 \mathrm{~Hz}, 1 \mathrm{H}), 3.88(\mathrm{dd}, J=16.8$, $2.0 \mathrm{~Hz}, 1 \mathrm{H}), 3.54-3.62(\mathrm{~m}, 2 \mathrm{H}), 2.51(\mathrm{~d}, J=12.0 \mathrm{~Hz}, 1 \mathrm{H}), 2.41(\mathrm{~s}, 3 \mathrm{H}), 2.35$ (ddd, $J=11.7,9.1$, $3.1 \mathrm{~Hz}, 1 \mathrm{H}), 1.99-2.03(\mathrm{~m}, 1 \mathrm{H}), 1.75-1.81(\mathrm{~m}, 2 \mathrm{H}), 1.46-1.52(\mathrm{~m}, 1 \mathrm{H}), 1.20-1.29(\mathrm{~m}, 3 \mathrm{H}) ;{ }^{13} \mathrm{C}-\mathrm{NMR}$ $\left(100 \mathrm{MHz}, \mathrm{CDCl}_{3}\right) \delta 200.5,144.5,134.9,134.0,133.3,131.5,130.3,130.0,128.7,128.5,127.5,127.4$, 126.6, 124.4, 88.7, 80.4, 64.5, 48.9, 29.7, 29.1, 23.9, 23.8, 21.6; HRMS (ESI+) calcd for $\left[\mathrm{C}_{26} \mathrm{H}_{27} \mathrm{NO}_{4} \mathrm{~S}\right]^{+}: m / z 450.1734$, found 450.1732 . 
1-(Thien-2-yl)-2-\{(2S,3aS,7aS)-3-tosyloctahydrobenzo[d]oxazol-2-yl\}ethanone (7k). 25\% yield; brown solid; IR $\left(\mathrm{CH}_{2} \mathrm{Cl}_{2}\right) v_{\max } 2943.0,1659.0,1598.0,1350.8,1162.2 \mathrm{~cm}^{-1} ;{ }^{1} \mathrm{H}-\mathrm{NMR}\left(400 \mathrm{MHz}, \mathrm{CDCl}_{3}\right)$ $\delta 7.80(\mathrm{dd}, J=3.8,1.0 \mathrm{~Hz}, 1 \mathrm{H}), 7.69(\mathrm{~d}, J=8.4 \mathrm{~Hz}, 2 \mathrm{H}), 7.65(\mathrm{dd}, J=5.0,1.0 \mathrm{~Hz}, 1 \mathrm{H}), 7.35(\mathrm{~d}$, $J=8.0 \mathrm{~Hz}, 2 \mathrm{H}), 7.14(\mathrm{dd}, J=5.0,3.8 \mathrm{~Hz}, 1 \mathrm{H}), 5.51(\mathrm{dd}, J=9.2,2.0 \mathrm{~Hz}, 1 \mathrm{H}), 3.63$ (dd, $J=16.4$, $2.0 \mathrm{~Hz}, 1 \mathrm{H}), 3.54$ (ddd, $J=10.9,8.9,2.9 \mathrm{~Hz}, 1 \mathrm{H}), 3.44(\mathrm{dd}, J=16.2,8.6 \mathrm{~Hz}, 1 \mathrm{H}), 2.41-2.49(\mathrm{~m}, 4 \mathrm{H})$, 2.31 (ddd, $J=12.2,8.6,3.0 \mathrm{~Hz}, 1 \mathrm{H}), 1.97-1.99(\mathrm{~m}, 1 \mathrm{H}), 1.75-1.81(\mathrm{~m}, 2 \mathrm{H}), 1.44-1.50(\mathrm{~m}, 1 \mathrm{H})$, $1.16-1.27(\mathrm{~m}, 3 \mathrm{H}) ;{ }^{13} \mathrm{C}-\mathrm{NMR}\left(100 \mathrm{MHz}, \mathrm{CDCl}_{3}\right) \delta 189.4,144.5,144.0,134.2,132.6,131.5,130.0$, 128.3, 88.3, 80.4, 64.4, 46.4, 29.6, 29.0, 23.8, 23.3, 21.6; HRMS (ESI+) calcd for $\left[\mathrm{C}_{20} \mathrm{H}_{23} \mathrm{NO}_{4} \mathrm{~S}_{2}\right]^{+}: \mathrm{m} / z$ 406.1141 , found 406.1136 .

(2S,4S)-4-Isopropyl-3-tosyl-2-(tosylmethyl)oxazolidine (8a). 30\% yield; white solid; IR $\left(\mathrm{CH}_{2} \mathrm{Cl}_{2}\right) v_{\max }$ 3064.1, 2963.9, 1597.1, 1350.3, 1318.9, $1163.7 \mathrm{~cm}^{-1}$; ${ }^{1} \mathrm{H}-\mathrm{NMR}\left(400 \mathrm{MHz}, \mathrm{CDCl}_{3}\right) \delta 7.82(\mathrm{~d}$, $J=8.4 \mathrm{~Hz}, 2 \mathrm{H}), 7.53(\mathrm{~d}, J=8.4 \mathrm{~Hz}, 2 \mathrm{H}), 7.37(\mathrm{~d}, J=8.0 \mathrm{~Hz}, 2 \mathrm{H}), 7.30(\mathrm{~d}, J=8.0 \mathrm{~Hz}, 2 \mathrm{H}), 5.13(\mathrm{dd}$, $J=8.8,1.2 \mathrm{~Hz}, 1 \mathrm{H}), 3.90(\mathrm{dd}, J=14.2,1.4 \mathrm{~Hz}, 1 \mathrm{H}), 3.74(\mathrm{dd}, J=9.4,2.2 \mathrm{~Hz}, 1 \mathrm{H}), 3.34-3.44(\mathrm{~m}, 2 \mathrm{H})$, $3.07(\mathrm{dd}, J=9.2,6.0 \mathrm{~Hz}, 1 \mathrm{H}), 2.46(\mathrm{~s}, 3 \mathrm{H}), 2.42(\mathrm{~s}, 3 \mathrm{H}), 1.78-1.87(\mathrm{~m}, 1 \mathrm{H}), 0.94(\mathrm{~d}, J=6.8 \mathrm{~Hz}, 3 \mathrm{H})$, $0.87(\mathrm{~d}, J=6.8 \mathrm{~Hz}, 3 \mathrm{H}) ;{ }^{13} \mathrm{C}-\mathrm{NMR}\left(100 \mathrm{MHz}, \mathrm{CDCl}_{3}\right) \delta 145.0,136.6,132.9,130.1,129.9,128.3$, 128.1, 128.0, 86.7, 68.4, 64.4, 61.6, 31.3, 21.7, 19,4, 18.2; HRMS (ESI+) calcd for $\left[\mathrm{C}_{21} \mathrm{H}_{27} \mathrm{NO}_{5} \mathrm{~S}_{2}\right]^{+}$: $\mathrm{m} / \mathrm{z} 438.1403$, found 438.1387 .

(2S,4S)-4-Methyl-3-tosyl-2-(tosylmethyl)oxazolidine (8b). $68 \%$ yield; white oil; IR $\left(\mathrm{CH}_{2} \mathrm{Cl}_{2}\right) v_{\max }$ 3063.2, 2983.5, 1597.6, 1351.3, $1164.6 \mathrm{~cm}^{-1}$; ${ }^{1} \mathrm{H}-\mathrm{NMR}\left(400 \mathrm{MHz}, \mathrm{CDCl}_{3}\right) \delta 7.80$ (d, $\left.J=8.4 \mathrm{~Hz}, 2 \mathrm{H}\right)$, $7.56(\mathrm{~d}, J=13.6 \mathrm{~Hz}, 2 \mathrm{H}), 7.35(\mathrm{~d}, J=8.0 \mathrm{~Hz}, 2 \mathrm{H}), 7.28(\mathrm{~d}, J=8.0 \mathrm{~Hz}, 2 \mathrm{H}), 5.17(\mathrm{~d}, J=9.2 \mathrm{~Hz}, 1 \mathrm{H})$, $3.85(\mathrm{~d}, J=14.4 \mathrm{~Hz}, 1 \mathrm{H}), 3.63(\mathrm{ddd}, J=11.1,6.7,2.7 \mathrm{~Hz}, 1 \mathrm{H}), 3.36-3.48(\mathrm{~m}, 3 \mathrm{H}), 2.43(\mathrm{~s}, 3 \mathrm{H}), 2.40$ $(\mathrm{s}, 3 \mathrm{H}), 1.25(\mathrm{~d}, J=6.4 \mathrm{~Hz}, 3 \mathrm{H}) ;{ }^{13} \mathrm{C}-\mathrm{NMR}\left(100 \mathrm{MHz}, \mathrm{CDCl}_{3}\right) \delta 145.0,144.7,136.7,132.7,130.1$, 129.9, 128.3, 127.8, 86.8, 73, 61.6, 54.8, 21.7, 21.6, 20.7; HRMS (ESI+) calcd for $\left[\mathrm{C}_{19} \mathrm{H}_{23} \mathrm{NO}_{5} \mathrm{~S}_{2}\right]^{+}$: $\mathrm{m} / \mathrm{z} 410.1090$, found 410.1094 .

(2S,4S)-4-Benzyl-3-tosyl-2-(tosylmethyl)oxazolidine (8c). 65\% yield; yellow oil; IR $\left(\mathrm{CH}_{2} \mathrm{Cl}_{2}\right) v_{\max }$ 3062.4, 3029.4, 2927.1, 1597.4, 1352.2, $1163.6 \mathrm{~cm}^{-1}$; ${ }^{1} \mathrm{H}-\mathrm{NMR}\left(400 \mathrm{MHz}, \mathrm{CDCl}_{3}\right) \delta 7.77(\mathrm{~d}$, $J=8.4 \mathrm{~Hz}, 2 \mathrm{H}), 7.59(\mathrm{~d}, J=8.0 \mathrm{~Hz}, 2 \mathrm{H}), 7.15-7.37(\mathrm{~m}, 9 \mathrm{H}), 5.18(\mathrm{dd}, J=9.2,1.6 \mathrm{~Hz}, 1 \mathrm{H}), 3.73-3.81$ (m, 2H), $3.66(\mathrm{dd}, J=9.4,3.4 \mathrm{~Hz}, 1 \mathrm{H}), 3.22(\mathrm{dd}, J=9.2,6.4 \mathrm{~Hz}, 1 \mathrm{H}), 3.08(\mathrm{dd}, J=14.6,9.4 \mathrm{~Hz}, 1 \mathrm{H})$, $3.02(\mathrm{dd}, J=13.6,3.2 \mathrm{~Hz}, 1 \mathrm{H}), 2.84(\mathrm{dd}, J=13.4,9.4 \mathrm{~Hz}, 1 \mathrm{H}), 2.46(\mathrm{~s}, 3 \mathrm{H}), 2.42(\mathrm{~s}, 3 \mathrm{H}) ;{ }^{13} \mathrm{C}-\mathrm{NMR}$ $\left(100 \mathrm{MHz} \mathrm{CDCl}_{3}\right) \delta 144.9,136.7,136.5,132.8,130.2,129.8,128.7,128.3,127.8,127.0,86.9,69.5$, 61.0, 59.8, 40.4, 21.7, 21.6; HRMS (ESI+) calcd for $\left[\mathrm{C}_{25} \mathrm{H}_{27} \mathrm{NO}_{5} \mathrm{~S}_{2}\right]^{+}: m / z$ 486.1403, found 486.1403.

[(2S,4R)-3-Tosyl-2-(tosylmethyl)oxazolidin-4-yl]methanol (8d). 81\% yield; white oil; IR $\left(\mathrm{CH}_{2} \mathrm{Cl}_{2}\right) v_{\max }$ 3532.6, 3063.3, 2928.6, 1597.3, 1351.3, $1164.0 \mathrm{~cm}^{-1}$; ${ }^{1} \mathrm{H}-\mathrm{NMR}\left(400 \mathrm{MHz}, \mathrm{CDCl}_{3}\right) \delta 7.78(\mathrm{~d}$, $J=8.0 \mathrm{~Hz}, 2 \mathrm{H}), 7.58(\mathrm{~d}, J=8.4 \mathrm{~Hz}, 2 \mathrm{H}), 7.33(\mathrm{~d}, J=8.0 \mathrm{~Hz}, 2 \mathrm{H}), 7.30$ (d, $J=8.0 \mathrm{~Hz}, 2 \mathrm{H}), 5.24(\mathrm{~d}$, $J=8.8 \mathrm{~Hz}, 1 \mathrm{H}), 3.76-3.83(\mathrm{~m}, 2 \mathrm{H}), 3.53-3.65(\mathrm{~m}, 4 \mathrm{H}), 3.38(\mathrm{dd}, J=9.2,6.4 \mathrm{~Hz}, 1 \mathrm{H}), 2.82(\mathrm{~s}, 1 \mathrm{H})$, $2.42(\mathrm{~s}, 3 \mathrm{H}), 2.41(\mathrm{~s}, 3 \mathrm{H}) ;{ }^{13} \mathrm{C}-\mathrm{NMR}\left(100 \mathrm{MHz}, \mathrm{CDCl}_{3}\right) \delta 145.1,145.0,136.6,132.4,130.3,13.2$, $129.9,129.8,128.2,127.9,87.1,68.0,62.8,60.9,59.7,21.7,21.6$; HRMS (ESI+) calcd for $\left[\mathrm{C}_{19} \mathrm{H}_{23} \mathrm{NO}_{6} \mathrm{~S}_{2}\right]^{+}: \mathrm{m} / z$ 426.1040, found 426.1042 . 
(2S,3aS,7aS)-3-Tosyl-2-(tosylmethyl)octahydrobenzo[d]oxazole (8e). 37\% yield; white oil; IR $\left(\mathrm{CH}_{2} \mathrm{Cl}_{2}\right) v_{\max } 3056.0,2942.9,1597.4,1352.8,1161.4 \mathrm{~cm}^{-1} ;{ }^{1} \mathrm{H}-\mathrm{NMR}\left(400 \mathrm{MHz}, \mathrm{CDCl}_{3}\right) \delta 7.82(\mathrm{~d}$, $J=8.4 \mathrm{~Hz}, 2 \mathrm{H}), 7.44(\mathrm{~d}, J=8.4 \mathrm{~Hz}, 2 \mathrm{H}), 7.36(\mathrm{~d}, J=8.0 \mathrm{~Hz}, 2 \mathrm{H}), 7.29(\mathrm{~d}, J=8.0 \mathrm{~Hz}, 2 \mathrm{H}), 5.13(\mathrm{dd}$, $J=9.4,1.4 \mathrm{~Hz}, 1 \mathrm{H}), 3.83(\mathrm{dd}, J=14.8,1.6 \mathrm{~Hz}, 1 \mathrm{H}), 3.54(\mathrm{dd}, J=14.8,9.6 \mathrm{~Hz}, 1 \mathrm{H}), 3.30$ (ddd, $J=11.8,8.2,2.0 \mathrm{~Hz}, 1 \mathrm{H}), 2.45$ (s, 3H), 2.42 (s, 3H), 2.19-2.27 (m, 1H), 1.71-1.85 (m, 4H), 1.33-1.43 $(\mathrm{m}, 1 \mathrm{H}), 1.15-1.24(\mathrm{~m}, 3 \mathrm{H}) ;{ }^{13} \mathrm{C}-\mathrm{NMR}\left(100 \mathrm{MHz}, \mathrm{CDCl}_{3}\right) \delta 144.8,136.8,130.9,130.3,130.0,128.4$, 86.0, 80.8, 64.1, 60.7, 29.7, 29.5, 23.6, 23.2, 21.6; HRMS (ESI+) calcd for $\left[\mathrm{C}_{22} \mathrm{H}_{27} \mathrm{NO}_{5} \mathrm{~S}_{2}\right]^{+}: \mathrm{m} / \mathrm{z}$ 450.1403 , found 450.1406 .

\section{Conclusions}

We have generated a chemical library of 60 unique oxazolidines, which will be tested for biological activity. We have also expanded the scope of the possible pro-nucleophiles and electron-deficient acetylenes that can be employed in mixed double-Michael reactions, thereby enabling the syntheses of more-diverse oxazolidines.

\section{Acknowledgments}

This project was supported by grants (R01GM071779 and P41GM081282) from the U.S. National Institutes of Health (NIH). We thank Greg Khitrov for mass spectrometric analyses. Funding for the LCMS system was provided by the National Center for Research Resources (S10RR025631).

\section{References and Notes}

1. For review, see: Katritzky, A.R., Ed. Advances in Heterocyclic Chemistry; Academic Press: London, UK, 2011; Volume 102 (and previous volumes).

2. For another review, see: Gribble, G.W., Joule, J.A., Eds. Progress in Heterocyclic Chemistry; Elsevier: Oxford, UK, 2011; Volume 22 (and previous volumes).

3. Dolle, R.E.; Le Bourdonnec, B.; Worm, K.; Morales, G.A.; Thomas, C.J.; Zhang, W. Comprehensive Survey of Chemical Libraries for Drug Discovery and Chemical Biology. J. Comb. Chem. 2010, 12, 765-806.

4. Terret, N.K. Combinatorial Chemistry; Oxford University Press: Oxford, UK, 1998.

5. Schreiber, S.L. Target-Oriented and Diversity-Oriented Organic Synthesis in Drug Discovery. Science 2000, 287, 1964-1969.

6. Nielsen, T.E.; Schreiber, S.L. Towards the Optimal Screening Collection: A Synthesis Strategy. Angew. Chem. Int. Ed. Engl. 2008, 47, 48-56.

7. Galloway, W.R.J.D.; Isidro-Llobet, A.; Spring, D.R. Diversity-oriented synthesis as a tool for the discovery of novel biologically functional small molecules. Nat. Commun. 2010, 1, 80-92.

8. Schreiber, S.L., Kapoor, T.M., Wess, G., Eds. Chemical Biology: From Small Molecules to Systems Biology and Drug Design; Wiley-VCH: Weinheim, Germany, 2008.

9. Strausberg, R.L.; Schreiber, S.L. From knowing to controlling: A path from genomics to drugs using small molecule probes. Science 2003, 300, 294-295.

10. Hopkins, A.L.; Groom, C.R. The druggable genome. Nat. Rev. Drug Discov. 2002, 1, 727-730. 
11. Zhu, X.; Lan, J.; Kwon, O. An Expedient Phosphine-Catalyzed [4 + 2] Annulation: Synthesis of Highly Functionalized Tetrahydropyridiness. J. Am. Chem. Soc. 2003, 125, 4716-4717.

12. Zhu, X.; Henry, C.E.; Kwon, O. A highly diastereoselective synthesis of 3-carbethoxy-2,5disubstituted-3-pyrrolines by phosphine catalysis. Tetrahedron 2005, 61, 6276-6282.

13. Zhu, X.; Henry, C.E.; Wang, J.; Dudding, T.; Kwon, O. A Phosphine-Catalyzed Synthesis of 1,3Dioxan-4-ylidenes. Org. Lett. 2005, 7, 1387-1390.

14. Zhu, X.; Schaffner, A.; Li, R.C.; Kwon, O. Phosphine-Catalyzed Synthesis of 6-Substituted 2-Pyrones: Manifestation of E/Z-Isomerism in the Zwitterionic Intermediate. Org. Lett. 2005, 7, 2977-2980.

15. Castellano, S.; Fiji, H.D.G.; Kinderman, S.S.; Watanabe, M.; De Leon, P.; Tamanoi, F.; Kwon, O. Small-Molecule Inhibitors of Protein Geranylgeranyltransferase Type I. J. Am. Chem. Soc. 2007, 129, 5843-5845.

16. Henry, C.E.; Kwon, O. Phosphine-Catalyzed Synthesis of Highly Functionalized Coumarins. Org. Lett. 2007, 9, 3069-3072.

17. Tran, Y.S.; Kwon, O. Phosphine-Catalyzed [4+2] Annulation: Synthesis of Cyclohexenes. J. Am. Chem. Soc. 2007, 129, 12632-12633.

18. Creech, G.S.; Kwon, O. Alcohol-Assisted Phosphine Catalysis: One-Step Synthesis of Dihydropyrone from Aldehyde and Allenoate. Org. Lett. 2008, 10, 429-432.

19. Watanabe, M.; Fiji, H.D.G.; Guo, L.; Umetsu, A.; Kinderman, S.S.; Slamon, D.J.; Kwon, O.; Tamanoi, F. Inhibitors of protein geranylgeranyltransferase-I and Rab geranylgeranyltransferase identified from a library of allenoate derived compounds. J. Biol. Chem. 2008, 283, 9571-9579.

20. Creech, G.S.; Zhu, X.; Fonovic, B.; Dudding, T.; Kwon, O. Theory-Guided Design of Brønsted Acid-Assisted Phosphine Catalysis: Synthesis of Dihydropyrones from Aldehydes and Allenoates Tetrahedron 2008, 64, 6935-6942.

21. Lu, J.; Chan, L.; Fiji, H.D.G.; Dahl, R.; Kwon, O.; Tamanoi, F. In vivo anticancer effect of a novel inhibitor of protein geranylgeranyltransferase I. Mol. Cancer Ther. 2009, 8, 1218-1226.

22. Guo, H.; Xu, Q.; Kwon, O. Phosphine-Promoted [3+3] Annulation of Aziridines with Allenoates: A Facile Enrty into Highly Functionalized Tetrahydropyridines. J. Am. Chem. Soc. 2009, 131, 6318-6319.

23. Lu, K.; Kwon, O. Phosphine-catalyzed [4+2] Annulation: Synthesis of Ethyl 6-phenyl-1-tosyl1,2,5,6-tetrahydropyridine-3-carboxylate. Org. Synth. 2009, 86, 212-224.

24. Khong, S.N.; Tran, Y.S.; Kwon, O. Equilibria Between Vinylogous Ylides and Phosphonium Dienolate Zwitterions: Vinylogous Wittig Olefination Versus Vinylogous Aldol-Type Reactions. Tetrahedron 2010, 66, 4760-4768.

25. Wang, Z.; Castellano, S.; Kinderman, S.S.; Argueta, C.E.; Beshir, A.B.; Fenteany, G.; Kwon, O. Diversity via a Branched Reaction Pathway: Generation of A Library of Sixteen Muticyclic Scaffolds and Identification of Anti-Migratory Agents. Chem. Eur. J. 2011, 17, 649-654.

26. Andrews, I.P.; Kwon, O. Phosphine Catalyzed [3+2] Annulation: Synthesis of Ethyl 5-(tertButyl)-2-phenyl-1-Tosyl-3-Pyrroline-3-Carboxylate. Org. Synth. 2011, 88, 138-151.

27. Choi, J.; Mouillesseauex, K.; Wang, Z.; Fiji, H.D.G.; Kinderman, S.S.; Otto, G.W.; Geisler, R.; Kwon, O.; Chen, J.-N. Alplexone Targets the HMG-CoA Reductase Pathway and Differentially Regulates Arteriovenous Angiogenesis. Development 2011, 138, 1173-1182. 
28. Cruz, D.; Wang, Z.; Kibbie, J.; Modlin, R.; Kwon, O. Diversity through phosphine catalysis identifies octahydro-1,6-naphthyridin-4-ones as activators of endothelium-driven innate immunity. Proc. Natl. Acad. Sci. USA 2011, 108, 6769-6774.

29. Martin, T.J.; Tran, Y.S.; Vakhshori, V.G.; Kwon, O. Phosphine-Catalyzed $\beta^{\prime}$-Umpolung Addition of Activated $\alpha$-Alkyl Allenes. Org. Lett. 2011, DOI:10.1021/o1200697m.

30. Vardhineedi, S.; Barcan, G.A.; Kwon, O. Bisphosphine-Catalyzed Mixed Double-Michael Reactions: Asymmetric Synthesis of Oxazolidines, Thiazolidines, and Pyrrolidines. J. Am. Chem. Soc. 2007, 129, 12928-12929.

31. Vardhineedi, S.; Kwon, O. Diphosphine-Catalyzed Mixed Double-Michael Reaction: A Unified Synthesis of Indolines, Dihydropyrrolopyridines, Benzimidazolines, Tetrahydroquinolines, Tetrahydroisoquinolines, Dihydrobenzo-1,4-oxazines, and Dihydrobenzo-3,1-oxazines. Org. Lett. 2010, 12, 1084-1087.

32. White, D.A.; Baizer, M.M. Michael additions catalyzed by phosphines. An overlooked synthetic method. Tetrahedron Lett. 1973, 14, 3597-3600.

33. Inanaga, J.; Baba, Y.; Hanamoto, T. Organic Synthesis with Trialkylphosphine Catalysts. Conjugate Addition of Alcohols to $\alpha, \beta$-Unsaturated Alkynic Acid Esters. Chem. Lett. 1993, 2, 241-244.

34. Grossman, R.B.; Pendharkar, D.S.; Patrick, B.O. $[n+1]$ Annulation Route to Highly Substituted Cyclic Ketones with Pendent Ketone, Nitrile, and Ester Functionality. J. Org. Chem. 1999, 64, 7178-7183.

35. Grossan, R.B.; Comesse, S.; Rasne, R.M.; Hattori, K.; Delong, M.N. Phosphoramidites Are Efficient, Green Organocatalysts for the Michael Reaction. Mechanistic Insights into the Phosphorous-Catalyzed Michael Reaction of Alkynones and Implications for Asymmetric Catalysis. J. Org. Chem. 2003, 68, 871-874.

36. Yavari, I.; Souri, S.; Sirouspour, M.; Djahaniani, H. Vinylphosphonium Salt Mediated Reaction between Alkyl Propiolates and Aminophenols or Hydroxyphenols. Synthesis 2006, 3243-3249.

37. Dithianes can be formed through the double-Michael reaction of propane 1,3-dithiol in the presence of bases (e.g., NaOMe, NaOEt, $\mathrm{NBu}_{4} \mathrm{OH}$ ); see: Gaunt, M.J.; Sneddon, H.F.; Hewitt, P.R.; Orsini, P.; Hook, D.F.; Ley, S.V. Development of $\beta$-keto 1,3-dithianes as versatile intermediates for organic synthesis. Org. Bimol. Chem. 2003, 1, 15-16.

38. Scott, J.D.; Williams, R.M. Total Synthesis of (-)-Tetrazomine. Determination of the Stereochemistry of Tetrazomine and the Synthesis and Biological Activity of tetrazomine Analogues. J. Am. Chem. Soc. 2002, 124, 2951-2956.

39. Herberich, B.; Scott, J.D.; Williams, R.M. Synthesis of a netropsin conjugate of a water-soluble epi-quinocarcin analogue: The importance of stereochemistry at nitrogen. Bioorg. Med. Chem. 2000, 8, 523-532.

40. Jacobsen, E.N., Pfaltz, A., Yamamoto, H., Eds. Comprehensive Asymmetric Catalysis I-III, 2nd ed.; Springer: Berlin, Germany, 1999.

41. Ojima, I., Ed. Asymmetric Synthesis; VCH: New York, NY, USA, 2000.

42. Fache, F.; Schultz, E.; Tommasino, M.L.; Lemaire, M. Nitrogen-Containing Ligands for Asymmetric Homogeneous and Heterogeneous Catalysis. Chem. Rev. 2000, 100, 2159-2232.

43. Paquette, L.A., Ed. Chiral Reagents for Asymmetric Synthesis; Wiley: Chichester, UK, 2003. 
44. Seayad, J.; List, B. Asymmetric organocatalysis. Org. Biomol. Chem. 2005, 3, 719-724.

45. Wolf, C.; Xu, H. Asymmetric catalysis with chiral oxazolidine ligands. Chem. Commun. 2011, 47, 3339-3350.

46. Characterization of 1d: Choi, J.Y.; Borch, R.F. Highly Efficient Synthesis of Enantiomerically Enriched 2-Hydroxymethylaziridines by Enzymatic Desymmetrization. Org. Lett. 2007, 9, 215-218.

47. Pei, Y.; Brade, K.; Brulé, E.; Hagber, L.; Lake, F.; Moberg, C. A General Method for the Preparation of Chiral TREN Derivatives. Eur. J. Org. Chem. 2005, 2005, 2835-2840.

48. Berry, M.B.; Craig, D. A Convenient Method for the Preparation of Enantiomerically Pure 2-Substituted $N$-Tosylaziiridines. Synlett 1992, 41-44.

49. Wang, Z.; Cui, Y.-T.; Xu, Z.-B.; Qu, J. Hot Water-Promoted Ring-Opening of Epoxides and Aziridines by Water and Other Nucleophiles. J. Org. Chem. 2008, 73, 2270-2274.

50. Yamashita, H. Metal(II) $d$-Tartrates Catalyzed Asymmetric Ring Opening of Oxiranes with Various Nucleophiles. Bull. Chem. Soc. Jpn. 1988, 61, 1213-1220.

51. Schiffers, I.; Rantanen, T.; Schmidt, F.; Bergmans, W.; Zani, L.; Bolm, C. Resolution of Racemic 2-Aminocyclohexanol Derivatives and Their Application as Lifands in Asymmetric Catalysis. J. Org. Chem. 2006, 71, 2320-2331.

52. Chen, P.C.; Wharton, R.E.; Patel, P.A.; Oyelere, A.K. Direct diazo-transfer reaction of $\beta$-lactam: Synthesis and preliminary biological activities of 6-triazolylpenicillanic acids. Bioorg. Med. Chem. 2007, 15, 7288-7300.

53. Calieno, G.; Fiorino, F.; Perissutti, E.; Severino, B.; Scolaro, D.; Gessi, S.; Cattabriga, E.; Borea, P.A.; Santagada, V. A convenient synthesis by microwave heating and pharmacological evaluation of novel benzoyltriazole and saccharine derivatives as $5-\mathrm{HT}_{1 \mathrm{~A}}$ receptor ligands. Eur. J. Pharm. Sci. 2002, 16, 15-28.

54. Chassaing, S.; Kueny-Stotz, M.; Isorez, G.; Brouillard, R. Rapid Preparation of 3Deoxyanthocyanidins and Novel Dicationic Derivatives: New Insight into an Old Procedure. Eur. J. Org. Chem. 2007, 38, 2438-2448.

55. Maeda, Y.; Kakiuchi, N.; Matsumura, S.; Nishimura, T.; Kawamura, T.; Uemura, S. Oxovanadium Complex-Catalyzed Aerobic Oxidation of Propargylic Alcohols. J. Org. Chem. 2002, 67, 6718-6724.

56. Ramachandran, P.V.; Rudd, M.T.; Reddy, M.V.R. Stereoselective synthesis of hex-2-(E)-en-4-yn1,6-dioates and E,Z-muconic acid diesters via organo-catalyzed self-coupling of propiolates. Tetrahedron Lett. 2005, 46, 2547-2549.

57. Balas, L.; Jousseaume, B.; Langwost, B. Improved preparation of aliphatic propynoic esters. Tetrahedron Lett. 1989, 30, 4525-526.

Sample Availability: Samples of compounds 3a-8e are available from the authors.

(C) 2011 by the authors; licensee MDPI, Basel, Switzerland. This article is an open access article distributed under the terms and conditions of the Creative Commons Attribution license (http://creativecommons.org/licenses/by/3.0/). 